\title{
Improvement of Polyunsaturated Fatty Acid Production in Echium acanthocarpum Transformed Hairy Root Cultures by Application of Different Abiotic Stress Conditions
}

\author{
Rafael Zárate, ${ }^{1}$ Elena Cequier-Sánchez, ${ }^{1,2}$ Covadonga Rodríguez, ${ }^{3,4}$ Roberto Dorta-Guerra, ${ }^{5}$ \\ Nabil El Jaber-Vazdekis, ${ }^{2}$ and Ángel G. Ravelo ${ }^{1,2}$ \\ ${ }^{1}$ Canary Islands Cancer Research Institute (ICIC), 61 Avenida La Trinidad, Torre A. Arévalo, 7th Floor, \\ 38204 La Laguna, Tenerife, Spain \\ ${ }^{2}$ Bio-Organic University Institute A.G. González, University of La Laguna, Ave. Fco. Sánchez, 38206 La Laguna, Tenerife, Spain \\ ${ }^{3}$ Animal Biology Deptartment (Physiology Unit), Biology Faculty, University of La Laguna, Ave. Fco. Sánchez, \\ 38206 La Laguna, Tenerife, Spain \\ ${ }^{4}$ Institute of Biomedical Technologies (ITB), University of La Laguna, Campus de Ofra, 38071 La Laguna, Tenerife, Spain \\ ${ }^{5}$ Statistics and Computation Deptartment, Maths Faculty, University of La Laguna, Ave. Fco. Sánchez, \\ 38206 La Laguna, Tenerife, Spain
}

Correspondence should be addressed to Rafael Zárate; rzarate@icic.es

Received 30 July 2013; Accepted 24 August 2013

Academic Editors: J. Contiero and E. Formentin

Copyright (C) 2013 Rafael Zárate et al. This is an open access article distributed under the Creative Commons Attribution License, which permits unrestricted use, distribution, and reproduction in any medium, provided the original work is properly cited.

\begin{abstract}
Fatty acids are of great nutritional, therapeutic, and physiological importance, especially the polyunsaturated n-3 fatty acids, possessing larger carbon chains and abundant double bonds or their immediate precursors. A few higher plant species are able to accumulate these compounds, like those belonging to the Echium genus. Here, the novel E. acanthocarpum hairy root system, which is able to accumulate many fatty acids, including stearidonic and $\alpha$-linolenic acids, was optimized for a better production. The application of abiotic stress resulted in larger yields of stearidonic and $\alpha$-linolenic acids, 60 and 35\%, respectively, with a decrease in linoleic acid, when grown in a nutrient medium consisting of B5 basal salts, sucrose or glucose, and, more importantly, at a temperature of $15^{\circ} \mathrm{C}$. The application of osmotic stress employing sorbitol showed no positive influence on the fatty acid yields; furthermore, the combination of a lower culture temperature and glucose did not show a cumulative boosting effect on the yield, although this carbon source was similarly attractive. The abiotic stress also influenced the lipid profile of the cultures, significantly increasing the phosphatidylglycerol fraction but not the total lipid neither their biomass, proving the appropriateness of applying various abiotic stress in this culture to achieve larger yields.
\end{abstract}

\section{Introduction}

Lipids in general and fatty acids (FA) in particular are essential metabolites displaying many key biological functions, acting as structural components of cell membranes, energy sources, and known intermediates in signaling pathways, besides their broad interest due to their important roles in human health and nutrition [1-6].

Oil producing plants could be an alternative dietary ingredient source of omega or $\mathrm{n}-3$ polyunsaturated fatty acids (PUFA) for the aquaculture industry; thus, it would be interesting to establish how the environmental factors modulate PUFA production in plants. These lack the ability to move to avoid possible environmental stress situations and, therefore, have to adapt to their environment in many different ways, and, similar to that described for fishes, temperature is one of the most influential environmental factors. Cold acclimation and the acquisition of freeze tolerance require the orchestration of many different seemingly disparate physiological and biochemical changes, including increasing sugar levels, soluble proteins, proline, certain organic acids, and new protein isoforms, alteration of lipidic 
membrane, and particularly differential expression of many genes coding for effector molecules that participate directly to alleviate stress [7].

Biological membranes are considered liquid crystals that behave as two-dimensional fluids. Thus, FA are able to participate in different adaptation mechanisms of plants to stress conditions, particularly through modification of the cell membrane fluidity and permeability. Furthermore, unsaturated FA facilitate the fluidity of lipids, having fewer van der Waals interactions, with the position of the double bonds in FA being more influential than their number [10]. Therefore, the addition of one or two double bonds to the membrane FA drastically decreases the transition temperature, and apparently the addition of a third or fourth double bond does not affect it. However, trienoic FA (e.g., 16:3 or 18:3) are the most abundant in chloroplast membranes, and increasing them allows a larger plant tolerance against low temperatures [11]. Inversely, in transgenic tobacco plants, in which the gene encoding chloroplast omega-3 fatty acid desaturase, which synthesizes trienoic FA, was silenced, displayed a lower level of trienoic FA than wild-type plants, offering a better acclimation to higher temperatures [12].

The PUFA dependence of membrane fluidity as well as of other physical properties including those necessary for membrane fusion events is a well-characterized phenomenon in animals including fish, fungi, bacteria, and plants [1317], together with the changes that occur when temperature increases or decreases. Unsaturated FA are thought to aid in maintaining membranes in a fluid state necessary for an appropriate biological functioning $[18,19]$.

In plants, FA such as $16: 0$ and $18: \ln -9$ are used to form lipid membranes by means of two different metabolic pathways: the chloroplast pathway or the cytoplasmic pathway, occurring in the endoplasmic reticulum (ER). In the first one, the FA of 16 carbons is generally esterified in the second position (sn-2) of the glycerol backbone, while in the ER within the synthesized lipids predominate those with 18 carbons. From here on, the desaturation process continues catalyzed by different enzymes which add an unsaturation to $18: 1 n-9$, resulting in $18: 2 n-6$, and so forth. Although there are differences between the desaturases found in chloroplasts (FAD7, FAD8) and those found in the ER (FAD2, FAD3) or in nonphotosynthetic tissues. The expression of each of their coding genes and enzymes is regulated by a complex system involving a variety of environmental stimuli, such as light or temperature, stress (damage, salt stress, pathogen invasion, etc.), or in response to the presence of jasmonate in the medium. Transgenic plants expressing or silencing enzymes of the biosynthetic pathway of PUFA have allowed the artificial modification of membrane FA and their physical and physiological adaptation to low temperatures. For instance, in Arabidopsis mutants deficient in one or more desaturase enzymes ( $\mathrm{fad}$ genes), the degree of FA unsaturation was extremely important in the plant response to cold temperatures [20], in particular in chloroplasts, where trienoic FA are important to ensure the correct biogenesis and maintenance of chloroplasts during plant growth at low temperatures [21].

Several authors have suggested that temperature is a factor capable of regulating even the expression of desaturase enzymes in several ways, such as transcription, posttranscription, and translational. In soybean seeds, low temperatures were applied and the data showed how D12 and D15 desaturases could be rapidly modulated in response to altered growth temperatures, while the enzymes for FA synthesis and elongation were not [22]. The regulation of these enzymes by temperature was documented with Brassica napus fad3 desaturase gene expressed in yeast; an increase in 18:3n-3 and FAD3 enzyme was recorded at low temperatures, but no fad 3 transcript was observed, suggesting that a posttranscriptional regulation was taking place [23].

Regarding the influence of osmotic stress on the FA profiles, osmotic pressure plays a crucial role as a regulator in the cellular water balance. Despite this important role, in in vitro plant culture systems employed for the production of secondary metabolites, much more attention has been applied to other factors, such as temperature or the nutrients present in the culture medium [24]. Few studies have addressed the use of osmotic stress or high osmotic pressure to stimulate the production of secondary metabolites in plant cell cultures. The application of osmotic stress on cells suspension cultures of Catharanthus roseus resulted in an increase in the intracellular accumulation of catharanthine and other alkaloids [25]. Other studies showed the influence of osmotic stress on anthocyanin production in cell culture of Vitis vinifera [26], in Populus deltoides [27], and in cell cultures of Panax notoginseng [28], and, more recently, it was applied in cell cultures of $C$. roseus [29], Taxus chinensis [30], and Panax ginseng [31], in order to induce the production of various alkaloids, paclitaxel, and saponins, respectively. In these studies, it has been confirmed that although occasionally the addition of sorbitol or mannitol can increase the production of metabolites of interest, it may also decrease the fresh weight of the cultures. Regarding the relationship between the induced stress and the production of PUFA, some studies have suggested that the hyperosmotic stress may reduce membrane fluidity similar to low temperatures. Contrary, the hypoosmotic stress effect is not well documented, but it has also been suggested that as the temperature is high, hypoosmotic stress may increase membrane fluidity.

Analogously, in the aquatic environment in general and in the field of fish aquaculture in particular, it is important to take into account environmental factors, especially temperature, a factor that directly influences the development and yield of crops. Cold affects fish growth and health and may decrease fish-farm production, even causing mortality through what is known as "winter syndrome" [32]. In some species adapted to very low temperatures, there is an interesting effect in order to keep the fluidity of cell membranes; they can decrease the chain length or increase the number of double bonds of those FA esterified in phospholipids [33]. Moreover, in deep-see living fish, it has been shown that high hydrostatic pressures exert the same influence as low temperatures, showing changes in certain enzyme activities, an increase in oxygen consumption, and increased membrane fluidity, achieved by increasing the unsaturation of FA esterified with phospholipids [33, 34]. 
In this study, we report the different strategies carried out in a novel Echium acanthocarpum transformed hairy root system, in order to particularly increase the production of the unusual PUFA, stearidonic (SDA; 18:4n-3) and $\gamma$-linolenic acids (GLA; 18:3n-6), of increasing pharmacological interest, by reducing culture temperature, applying osmotic stress, and changing the carbon source. Moreover, in order to determine the validation of the data and the effectiveness of the abiotic stress, a robust statistical approach was applied.

\section{Materials and Methods}

2.1. Plant Material. Seeds of E. acanthocarpum, donated by Jardín Botánico Viera y Clavijo, Gran Canaria, Spain, were first surface-sterilized by a brief immersion in $70 \% \mathrm{EtOH}$, followed by submersion in an aqueous solution of $5 \%(\mathrm{v} / \mathrm{v})$ of commercial bleach for $25 \mathrm{~min}$ with gentle hand agitation. They were finally washed 5 times with sterile distilled water.

Treated seeds were then germinated in vitro on a solid B5 [8] medium, supplemented with $3 \%$ sucrose, $3-4 \mathrm{mg} / \mathrm{L} \mathrm{GA3}$ (gibbereelic acid), and solidified with $0.7 \%$ agar, with the $\mathrm{pH}$ adjusted to 6.0 prior to autoclaving $\left(115^{\circ} \mathrm{C}, 1 \mathrm{~atm}\right.$. pressure, $15 \mathrm{~min}$.), contained in Petri dishes ( $90 \mathrm{~mm}$ diameter), and cultured in the dark until the beginning of germination. After germination, plants were transferred to the same solid nutrient medium without the addition of GA3, contained in translucent glass jars covered with a lid $(175 \mathrm{~mL}$ capacity, Sigma-Aldrich, MO, US), which were placed under light conditions ( $16 \mathrm{~h}$ photoperiod and irradiance of $35 \mathrm{mmol} \mathrm{m}^{2} \mathrm{~s}^{-1}$ supplied by cool white fluorescent tubes) and a temperature of $25 \pm 2^{\circ} \mathrm{C}$ to allow further plant growth.

Under sterile conditions, 50-60-day-old plants were employed for guided infection with Agrobacterium rhizogenes strain LBA1334 harboring a pBIN19-gus intron plasmid by repeatedly stabbing the internodal stem areas with a fine needle containing bacteria [35, 36]. After 25-30 days, hairy roots of 3-4 $\mathrm{mm}$ in length had developed and were aseptically excised and transferred to B5 liquid medium, containing the antibiotic cefotaxime $(100 \mathrm{mg} / \mathrm{L})$ as well as $1 \%$ of the antioxidant polyvinylpyrrolidone (PVP) for several subcultures. Finally, actively growing bacterium-free hairy roots were cut into small segments and routinely cultured and refreshed in Erlenmeyer flasks $(250 \mathrm{~mL})$, containing $30 \mathrm{~mL}$ of sterile B5 liquid medium supplemented with $3 \%$ sucrose and $1 \%$ of PVP (standard nutrient medium), sealed with a double layer of aluminum foil, and placed on an orbital shaker at $95 \mathrm{rpm}$ at $25 \pm 2^{\circ} \mathrm{C}$ in the dark.

For culture growth and FA production and analysis, different hairy root culture media of the established E1.5 cell line were investigated, each providing a particular abiotic stress and culture conditions (Table 1). In order to cover the entire growth period for each culture, sampling times were different since the kinetics of growth differed due mainly to the culture temperature; thus, the sampling points were as follows $\mathrm{T} 1,5$ days for culture $\mathrm{B} 1$ and 15 days for cultures $\mathrm{Cl}-$ C4; T2, 10 days for culture $\mathrm{B} 1$ and 25 days for cultures $\mathrm{C} 1-\mathrm{C} 4$; T3, 15 days for culture B1 and 35 days for cultures C1-C4; T4, 20 days for culture $\mathrm{B} 1$ and 45 days for cultures C1-C4; T5, 25 days for culture $\mathrm{B} 1$ and 65 days for cultures $\mathrm{Cl}-\mathrm{C} 4$; and T6, 35 days for culture $\mathrm{B} 1$ and 75 days for cultures $\mathrm{C} 1-\mathrm{C} 4$.

2.2. Lipid Extraction and Transesterification of Lipids. Hairy roots were separated from the liquid nutrient medium by vacuum filtration, weighed, and lyophilised at $-80^{\circ} \mathrm{C}$ for $24 \mathrm{~h}$ using a freeze-dryer (Christ Alpha 2-4, Osterode, Germany). Each sample was powdered using a mortar and pestle with liquid nitrogen. After homogenisation, total lipid was extracted following the method previously described [36-38].

Lipid aliquots $(2 \mathrm{mg})$ were subjected to acid-catalyzed transesterification by dissolving the sample in $1 \mathrm{~mL}$ toluene, employed to ensure that the neutral lipids got properly dissolved, plus $2 \mathrm{~mL}$ of a mixture of $\mathrm{MeOH} / 1 \% \mathrm{H}_{2} \mathrm{SO}_{4}$, and incubated in a capped glass test tube at $50^{\circ} \mathrm{C}$ for $16 \mathrm{~h}$ [39]. Prior to transmethylation, heneicosae-noic acid (21:0) (2.5\% of the total lipid analysed, $50 \mu \mathrm{g}$ ), was added as internal standard to the lipid extracts. Transesterification was conducted as previously described [36-38]. Preparative thin layer chromatography employing silica gel G-25 glass sheets (Macherey-Nagel, Germany), developed with a solvent system composed of hexane/diethyl ether/acetic acid 97.7\% $(90: 10: 1$, by vol) and visualized after brief sublimation of iodine with slight heat, was used for the isolation and purification of the fatty acids methyl esters (FAMEs). These ran close to the solvent front and were then scrapped off the glass sheet, extracted with $10 \mathrm{~mL}$ hexane/ethyl ether $(1: 1, \mathrm{v} / \mathrm{v})$, and dried under nitrogen. Finally, the samples were dissolved in $0.5-1.0 \mathrm{~mL}$ hexane and kept under nitrogen in sealed glass vials at $-20^{\circ} \mathrm{C}$ until analysis.

2.3. Gas Chromatography of FAMEs. Analysis and quantification of FAMEs were conducted by GC employing a Shimadzu GC-14A apparatus (Shimadzu, Japan) equipped with a flame ionization detector $\left(250^{\circ} \mathrm{C}\right)$, a Supelcowax 10 fused silica capillary column ( $30 \mathrm{~m} \times 0.32 \mathrm{~mm}$ ID), employing helium as carrier gas. Samples $(0.6 \mu \mathrm{L})$ were injected into the system by an on-column autoinjector (Shimadzu AOC-17) at $50^{\circ} \mathrm{C}$. A temperature program of $180^{\circ} \mathrm{C}$ for the first $10 \mathrm{~min}$, followed by an increase of $2.5^{\circ} \mathrm{C} / \mathrm{min}$ until reaching $215^{\circ} \mathrm{C}$, was employed for separation of the compounds.

FAMEs were identified according to their RT compared with standards of individual commercial FAMEs (linoleic acid methyl ester, methyl $\gamma$-linolenate, methyl oleate, stearidonic acid methyl ester, and heneicosanoic acid) and a wellcharacterized fish oil mix (Sigma-ref LUPE). They were quantified according to the amount of 21:0 added as internal standard prior to transmethylation and by comparison with a calibration curve created with the individual standards.

2.4. Statistical Analysis. Results are present as the means and standard deviations of three replicates for each sampling time for each of the treatments and cultures. The data were checked for normal distribution by one-sample KolmogorovSmirnov test as well as for homogeneity of the variance with the Levene test, and, when necessary, Bartlett test was also applied. When variance was not homogeneous, KruskalWallis and Games-Howele tests were conducted to assess 
TABLE 1: Liquid culture media employed for the growth and fatty acid studies of Echium acanthocarpum hairy roots grown under different stress conditions. B5 [8].

\begin{tabular}{lccccc}
\hline Name & Basal salt & Carbon source & PVP & Sorbitol & Temperature \\
\hline Culture B1 & B5 & $3 \%$ sucrose & $1 \%$ & - & - \\
Culture C1 & B5 & $3 \%$ sucrose & $1 \%$ & $1 \%$ & $0.2 \mathrm{M}$ \\
Culture C2 & B5 & $3 \%$ sucrose & $1 \%$ & - & $15^{\circ} \mathrm{C}$ \\
Culture C3 & B5 & $3 \%$ glucose & $1 \%$ & $0.2 \mathrm{M}$ & $15^{\circ} \mathrm{C}$ \\
Culture C4 & B5 & $3 \%$ glucose & & $15^{\circ} \mathrm{C}$ \\
\hline
\end{tabular}

PVP: polyvinyl pyrrolidone.

statistical differences. The effects of culture conditions and FA levels were firstly determined using one-way ANOVA test $(P<0.05)$. The percentages and total amounts of FA, particularly the contents of GLA and SDA in the different cultures, were included as variables in a principal component analysis (PCA). Principal components were subsequently analysed by two-way ANOVA to study the combined effects of both factors, FA profiles and stress conditions, as well as their interconnections. Statistical analyses were performed employing the SPSS software (versions 15.0 and 17.0, SPSS Inc., IL, USA).

\section{Results and Discussion}

Studies of growth conditions and type of stress applied to cultures were conducted in order to achieve FA profiles richer in $\Delta 6$-desaturated products, such as SDA and GLA.

\subsection{Effect of Stress on Echium acanthocarpum Hairy Roots} Growth. In order to carry out these experiments, hairy roots from line E1.5 were used taking into account the previously described results $[36,40]$. The data of growth were recorded under different nutrient media and conditions (cultures B1, $\mathrm{C} 1, \mathrm{C} 2, \mathrm{C} 3$, and C4).

A typical growth curve was achieved for all cultures, with a lag phase, which tended to be more pronounced in cultures $\mathrm{C} 2-\mathrm{C} 4$ than culture $\mathrm{C} 1$, at sampling points 1 and 2 (Figure 1). Then, the cultures started their exponential growth phase. Finally, the cultures described a stationary phase (points 4 and 5), and from that point on, cell death took place, in which cultures lost their hairy root morphology, showing also vitrification and browning.

In culture B1, a maximum fresh weight of $1.38 \mathrm{~g}$ was registered at sampling point 6 , whereas the maximum of culture $\mathrm{C} 1$ was $2.76 \mathrm{~g}$ at sampling point 5 . Statistical analysis of fresh weight of both cultures showed only significant difference for sampling points 1 and 2 (Figure 1).

In relation to the carbon source used, when glucose was used instead of sucrose, it would be expected that glucose would be able to induce a faster growth, particularly in the lag phase, since sucrose would require to be hydrolyzed into the monomers glucose and fructose to then be taken up by the tissues. However, both carbon sources displayed comparable growth rates (Figure 1). Thus, when fresh weight variation of hairy roots cultured at $15^{\circ} \mathrm{C}$ (cultures C1-C4) was compared, no significant differences were observed in

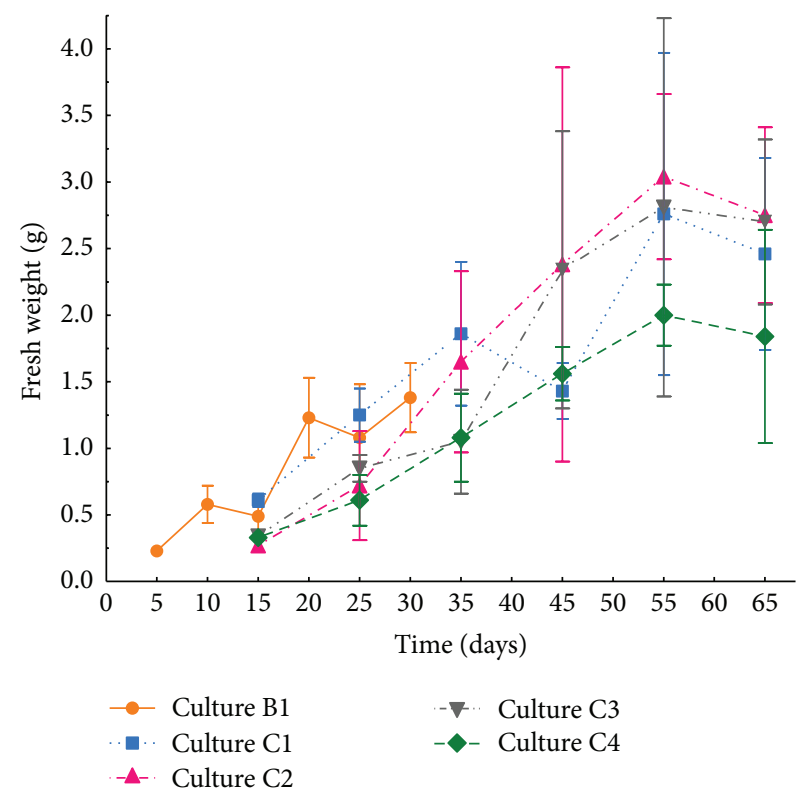

FIGURE 1: Fresh weight (g) variation of Echium acanthocarpum transformed hairy roots cell line E1.5 cultured in three different growth media and temperatures. Values represent the mean of three replicates $(n=3) \pm \mathrm{SD}$. Depending on the culture temperature $25^{\circ} \mathrm{C}$ (culture B1) or $15^{\circ} \mathrm{C}$ (cultures $\mathrm{C} 1-\mathrm{C} 4$ ), the sampling points varied as $T 1=5$ days for culture B1 and 15 days for cultures C1-C4; T2 = 10 days for culture B1 and 25 days for cultures $\mathrm{C} 1-\mathrm{C} 4 ; \mathrm{T} 3=15$ days for culture $\mathrm{B} 1$ and 35 days for cultures $\mathrm{C} 1-\mathrm{C} 4 ; \mathrm{T} 4=20$ days for culture $\mathrm{B} 1$ and 45 days for cultures $\mathrm{C} 1-\mathrm{C} 4 ; \mathrm{T} 5=25$ days for culture B1 and 55 days for cultures $\mathrm{C} 1-\mathrm{C} 4$; and $\mathrm{T} 6=30$ days for culture $\mathrm{B} 1$ and 65 days for cultures $\mathrm{C} 1-\mathrm{C} 4$.

any of the analyzed points, except for the first one (Figure 1). In culture $\mathrm{C} 2$, containing $0.2 \mathrm{M}$ sorbitol as osmotic pressure inducing agent, fresh weight reached $3.04 \mathrm{~g}$ at sampling point 5. Similarly, in cultures $\mathrm{C} 3$ and $\mathrm{C} 4$, the maximum values were recorded at sampling point 5, 2.81 and $2.00 \mathrm{~g}$, respectively, although no significant differences were observed in any of the sampling points (Figure 1). Interestingly, it has been described that the application of osmotic stress tends to decrease the fresh weight of in vitro cultured rice or wheat callus; this is mainly due to the accumulation of metabolites or osmolytes in the cytosol, such as glycinebetaine, proline, or soluble carbohydrates, which balance the effect of the osmotic pressure produced by the extracellular solute concentration, avoiding the massive loss of intracellular water [41-43]. This 
fact would not be present in E. acanthocarpum hairy roots, since no reduction in fresh weight was observed, but a rather slight increase. Taking into account the final fresh weight versus the initial fresh weight, culture $\mathrm{C} 2$ consisting of B5 mineral salts, $3 \%$ sucrose, $1 \%$ PVP and $0.2 \mathrm{M}$ sorbitol was the most effective, with a 12 -fold increase, whereas fresh weight of $\mathrm{C} 1, \mathrm{C} 3$, and $\mathrm{C} 4$ increased in the range of 8-11 folds. Nonetheless, a negative interaction of sorbitol on the growth of certain in vitro cultures, such as ginseng roots, with $3 \%$ sucrose was published. The data showed a considerable growth reduction when sorbitol $(0.2-0.3 \mathrm{M})$ was added [44]. However, a beneficial growth effect upon the addition of sorbitol $(54.97 \mathrm{mM})$ to a certain varieties of rice in vitro cultures has also been described [41]. It might be possible that sorbitol, a polyhydric alcohol sugar, could be metabolized by tissues when sucrose amounts get finished. In our study, the addition of sorbitol does not show any detrimental effect on growth but a rather slight nonsignificant increase.

3.2. Effect of Stress on Echium acanthocarpum Hairy Roots Lipid Composition: Principal Component Analyses. In general, the total lipid (TL) extracted from culture $\mathrm{C} 1$ hairy roots grown at $15^{\circ} \mathrm{C}$ was statistically similar to that of culture $\mathrm{B} 1$ grown at $25^{\circ} \mathrm{C}$ (Figure 2). The temperature did not significantly affect TL, and apparently E. acanthocarpum hairy roots are able to maintain homeostasis under these conditions. The data also indicate the possibility of further reducing the culture temperature to likely boosts its effect, on the production of desired $\Delta 6$-desaturated fatty acids, GLA, and SDA, as has been published for $B$. napus cultures grown at $4^{\circ} \mathrm{C}$ [45] or Arabidopsis grown at $12^{\circ} \mathrm{C}$ [46].

Analogously, the addition of sorbitol and/or glucose or both (cultures C2, C3, C4) did not seem to have a direct influence on TL since mostly no statistical differences were recorded except for sampling point 4 (Figure 2), although some reports showed that under water stress, total lipid clearly dropped in leaves of $A$. thaliana and Cocos nucifera $[47,48]$. The decrease in TL is often associated with the richness of saturated FA in the leaf, as they are a more accessible substrate for hydrolysis and peroxidation reactions [49] and also because the leaves contain chiefly monogalactosyl diacylglycerides (MGDG) and digalactosyl diacylglycerids (DGDG), which are mainly esterified with saturated FA under stress conditions. Therefore, some authors claim that chloroplasts and their membranes are the most sensitive plant organelle to water stress $[50,51]$ and low temperatures $[18,52,53]$. Given the nature of hairy roots, in which tissue cells with chlorophyll are not present, and therefore are not photosynthetic organs, could be the reason why $E$. acanthocarpum hairy roots seem not to be much affected by water or osmotic stress conditions, and the increment of TL at the initial sampling times might be due to a larger cell division rate in these meristematic tissues, accompanied by a major formation of cytoplasmic membranes and production of PUFA [54].

In order to study the effect of both decreasing the culture temperature from 25 to $15^{\circ} \mathrm{C}$ and the presence of other stress agents in E. acanthocarpum hairy roots, in lipid composition,

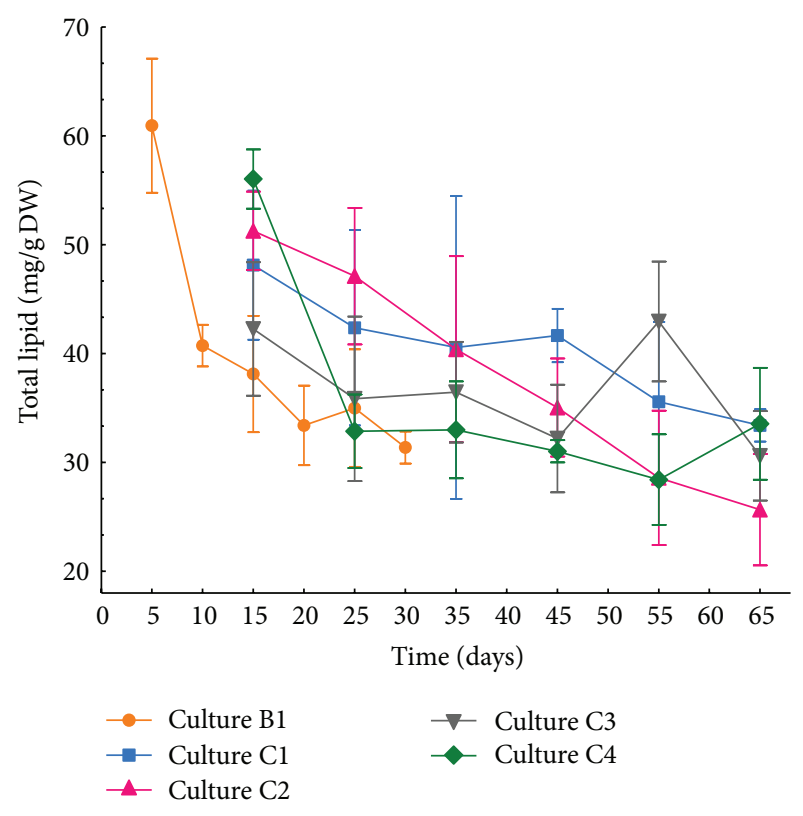

Figure 2: Total lipid (TL) content (mg/g DW) of E1.5 Echium acanthocarpum transformed hairy root cell line cultured in different culture media and temperatures. Values represent the mean of three independent replicates $(n=3) \pm \mathrm{SD}$. Depending on the growth temperature, the sampling points varied as $T 1,5$ days for culture B1 and 15 days for cultures C1-C4; T2, 10 days for culture B1 and 25 days for cultures $\mathrm{C} 1-\mathrm{C} 4$; T3, 15 days for culture $\mathrm{B} 1$ and 35 days for cultures C1-C4; T4, 20 days for culture B1 and 45 days for cultures $\mathrm{C} 1-\mathrm{C} 4$; T5, 25 days for culture $\mathrm{B} 1$ and 65 days for cultures $\mathrm{C} 1-\mathrm{C} 4$; and $T 6,35$ days for culture $\mathrm{B} 1$ and 75 days for cultures $\mathrm{C} 1-\mathrm{C} 4$.

sampling points 4 and 5 were taken as the most representative ones by making a compromise between biomass production and the rate of FA production [40]. Lipid class composition was affected by both, the temperature and the presence of sorbitol and glucose in the nutrient medium, although generally no significant differences were observed between the values of lipid classes PC, PS + PI, and PE and total polar and neutral lipids (Tables 2(a) and 2(b)). Among polar lipids, phosphatidylcholine (PC), phosphatidylserine, and phosphatidylinolsitol (PS + PI) ranged from 8.53 to $9.13 \%$ and 4.51 to $6.17 \%$ of the total lipids for PC and PS + PI, respectively, in cultures $\mathrm{B} 1$ and $\mathrm{Cl}$ (Table 2(a)). Different authors propose that under water stress conditions, free FA are produced most abundantly by the action of lipases over polar lipids and subsequently stored in esterified triacylglycerols (TAG), increasing thereby the neutral lipids. Furthermore, the polar lipids rise may be explained because water stress increases the synthesis of new membranes [55]. In the same fashion, it has been observed in roots grown at low temperatures how total content of polar lipids was augmented twice its initial value at room temperature $[56,57]$.

There was a clear increasing tendency of phosphatidylglycerol (PG) in E. acanthocarpum cultures grown at $15^{\circ} \mathrm{C}$, going from an average of $3.48 \%$ of $\mathrm{TL}$ at $25^{\circ} \mathrm{C}$ (culture $\mathrm{B} 1$, Table $2(\mathrm{a})$ ) to $8,25 \%$ of the $\mathrm{TL}$ at $15^{\circ} \mathrm{C}$ (culture $\mathrm{C} 1$, Table 2(a)). The rise of PG in E. acanthocarpum hairy roots 
TABLE 2: Total lipid (TL) amounts (mg/g DW) and lipid classes profile (\%) of Echium acanthocarpum hairy roots harvested at sampling points 4 and 5 growing under abiotic stress (low temperature in cultures $\mathrm{C} 1-\mathrm{C} 4$, osmotic stress in cultures $\mathrm{C} 2$ and $\mathrm{C} 4$, and glucose as carbon source in cultures $\mathrm{C} 3$ and $\mathrm{C} 4$ ).

(a) Data of sampling point 4

\begin{tabular}{lccccc}
\hline Sampling point 4 & Culture B1 & Culture C1 & Culture C2 & Culture C3 & Culture C4 \\
\hline TL (mg/g DW) & $33.41 \pm 3.66^{\mathrm{ab}}$ & $41.67 \pm 2.45^{\mathrm{b}}$ & $35.05 \pm 4.50^{\mathrm{ab}}$ & $32.20 \pm 4.94^{\mathrm{ab}}$ & $31.03 \pm 1.03^{\mathrm{a}}$ \\
PC & $9.30 \pm 1.37$ & $8.53 \pm 1.34$ & $6.46 \pm 1.81$ & $10.13 \pm 1.51$ & $6.50 \pm 1.18$ \\
PS + PI & $6.17 \pm 0.67$ & $4.51 \pm 4.09$ & $5.82 \pm 2.11$ & $6.92 \pm 2.09$ & $3.05 \pm 1.68$ \\
PG & $3.48 \pm 1.42$ & $8.25 \pm 1.70^{*}$ & $13.77 \pm 2.36$ & $9.12 \pm 2.32$ & $12.54 \pm 1.56$ \\
PE & $10.75 \pm 0.62$ & $12.18 \pm 1.49$ & $9.80 \pm 1.16$ & $11.02 \pm 0.77$ & $12.72 \pm 4.51$ \\
Polar lipid & $29.70 \pm 2.77$ & $33.49 \pm 5.93$ & $35.85 \pm 3.54$ & $37.19 \pm 2.89$ & $34.81 \pm 8.21$ \\
Neutral lipid & $64.05 \pm 5.67$ & $59.59 \pm 5.34$ & $59.97 \pm 2.44$ & $59.04 \pm 6.20$ & $65.19 \pm 8.21$ \\
unknown & $6.25 \pm 2.92$ & $6.92 \pm 2.21$ & $4.17 \pm 1.11$ & $3.77 \pm 3.68$ & $13.43 \pm 4.64$ \\
\hline
\end{tabular}

(b) Data of sampling point 5

\begin{tabular}{lccccc}
\hline Sampling point 5 & Culture B1 & Culture C1 & Culture C2 & Culture C3 & Culture C4 \\
\hline TL (mg/g DW) & & $35.58 \pm 7.33$ & $28.59 \pm 6.17$ & $42.96 \pm 5.50$ & $28.34 \pm 4.17$ \\
PC & - & $7.19 \pm 1.06$ & $5.72 \pm 1.64$ & $4.96 \pm 1.24$ & $8.54 \pm 0.97$ \\
PS + PI & - & $2.80 \pm 2.09$ & $6.27 \pm 2.35$ & $3.18 \pm 1.80$ & $5.09 \pm 1.45$ \\
PG & - & $5.12 \pm 0.70$ & $9.86 \pm 1.79$ & $9.96 \pm 2.23$ & $12.91 \pm 1.96$ \\
PE & - & $8.79 \pm 1.59$ & $9.32 \pm 1.47$ & $8.96 \pm 2.87$ & $13.97 \pm 1.95$ \\
Polar lipid & - & $23.91 \pm 5.42$ & $31.17 \pm 6.97$ & $27.06 \pm 4.92$ & $40.51 \pm 6.09$ \\
Neutral lipid & - & $71.97 \pm 7.95$ & $64.39 \pm 6.89$ & $72.94 \pm 4.92$ & $59.49 \pm 6.09$ \\
unknown & & $4.11 \pm 2.53$ & $4.43 \pm 0.21$ & $1.41 \pm 2.45$ & $12.30 \pm 1.69$ \\
\hline
\end{tabular}

${ }^{*}$ Significant differences $(P \leq 0.05)$ between cultures given by Student's $t$-test when comparing the mean of percentages of the lipid classes for cultures B1 and C1.

${ }^{\mathrm{a}, \mathrm{b}}$ Significant differences of TL values, also showing homogeneous subclusters as indicated by Tuckey's test when comparing the mean of TL values.

TL: total lipid; PC: phosphatidylcholine; PS + PI: sum of phosphatidylserine and phosphatidylinositol; PG: phosphatidylglycerol; PE: phosphatidylethanolamine.

Percentage values were transformed by the arcsin transformation prior to statistical analysis.

Values represent the mean of three independent experiments $(n=3) \pm \mathrm{SD}$.

Entry of culture B1 for sampling point 5 is empty because data were collected only for sampling 4.

in response to a reduction of temperature is consistent with several studies where the content of PG in roots of Avicennia germinans was higher than in those plants more resistant to low temperatures [58]. In another study carried out on the plasma membrane of wheat seedlings, a rise of all classes of phospholipids, including PG, was recorded when the culture temperature was $2^{\circ} \mathrm{C}$ [59]. It has also been speculated that the direct relationship between temperature and richness of PG in plant tissues is due to the glycerol-3P-acyltransferase specificity, responsible for the esterification of certain FA, usually 18:0, 18:1, and 16:0 at sn-1 position of PG and also due to its response to temperature $[60,61]$. No significant changes were observed in $\mathrm{PE}$ in $\mathrm{B} 1$ and $\mathrm{Cl}$ cultures, with percentages ranging from 10.75 to $12.18 \%$ of TL. Finally, when calculating the total values of polar and neutral lipids, no drastic changes were observed, with values of 29.70 to $33.39 \%$ and from 59.59 to $64.05 \%$, respectively (Table 2(a)). After studying the values for each lipid class in the other cultures (C2-C4), and compared to those profiles obtained in $\mathrm{C} 1$, no significant changes were detected, except for the case of PG, reaching $13.77 \%$ of TL in culture C2 (Tables 2 (a) and 2(b)). There are very few studies addressing the distribution of lipid classes in roots, although one describes the PG content in the thylakoid membranes and its relative increase, together with unsaturation enrichment of FA esterified in PG against low temperatures, both in monocotyledons and dicotyledons $[61,62]$. Also, it was reported that there is an enrichment of the saturated 16:0, 18:0 FA, constituting about $40 \%$ of those esterified FA in PG, and the monoene trans-16:1, in plant species sensitive to low temperatures, while the wealth of saturated FA in resistant species to low temperatures decreased to $20 \%$ [63]. These data suggest a direct relationship of this phospholipid with plant resistance to low temperatures, although other factors providing such feature might also exist [64].

The distribution of lipid classes in E. acanthocarpum hairy roots was similar to that of a nonphotosynthetic tissue, exhibiting high polar lipid values, mainly PE and PG, not recording the typical photosynthetic tissue lipids, such as monogalactosyl diacylglycerides (MGDG) and digalactosye diacylglycerides (DGDG), present in chloroplasts.

In order to carry out the statistical analysis of the data, variables for cultures $\mathrm{B} 1$ and $\mathrm{Cl}$, sharing the same culture medium, were analysed to observe how temperature (25 and $15^{\circ} \mathrm{C}$ ) affects the distribution of lipid classes. Only significant differences for PG were observed, whose percentage was the 


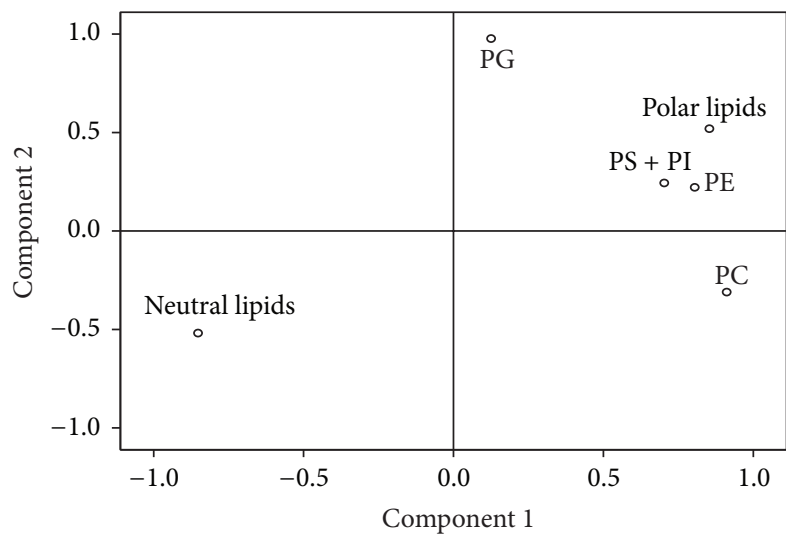

FIGURE 3: Factor loading plots of the principal component analyses of the percentages of lipid classes in Echium acanthocarpum E1.5 cell line hairy roots, growing in different media at $15^{\circ} \mathrm{C}(\mathrm{Cl}-\mathrm{C} 4$ cultures). $\mathrm{PC}=$ phosphatidylcholine; $\mathrm{PS}+\mathrm{PI}=$ phosphatidylserine and phosphatidylinositol; $\mathrm{PG}=$ phosphatidylglycerol; and $\mathrm{PE}=$ phosphatidylethanolamine.

highest when grown at $15^{\circ} \mathrm{C}$ (Tables $2(\mathrm{a})$ and $2(\mathrm{~b})$ ). Secondly, in order to study the influence of the use of glucose and $0.2 \mathrm{M}$ sorbitol in the nutrient medium and the time of sampling, a principal component analysis (PCA) to the percentages of the obtained lipid classes for $\mathrm{Cl}-\mathrm{C} 4$ cultures was performed (Table 3). We obtained two principal components ( $\mathrm{PCl}$ and PC2), which explained $85.68 \%$ of the variance. PC2 (19.78\% of variance) was positively correlated with the PG, while PC1 (65.91\% of variance) was positively related to PC, PS + PI, PE, and total polar lipids, while it was negatively correlated with the neutral lipids (Table 3, Figure 3).

Accordingly, it could be assumed that $\mathrm{PCl}$ is correlated with the richness of polar lipids and PC2 reflects the amount of PG in hairy root tissues.

After removal of the principal components $\mathrm{PCl}$ and PC2, we conducted a two-way ANOVA test in order to determine whether the studied factors, that is, stressing factors and sampling points and the interaction between them, had influence on the extracted new variables $\mathrm{PC} 1$ and PC2 (Table 4).

Only when both factors interacted for PCl, the level of significance was less than 0.05 (Table 4, Figure 4); that is, polar lipids usually remained constant. Conversely, the PC2 variable, reflecting the content of PG in the sample, (Figure 3, Table 3) was influenced by the presence of sorbitol or glucose in the nutrient medium (Table 4). Interestingly, it was observed that PG content was significantly higher in $\mathrm{C} 2-\mathrm{C} 4$ cultures, those in which $0.2 \mathrm{M}$ sorbitol or $3 \%$ glucose was present. These data are consistent to those observed in A. thaliana leaves reporting a nonsignificant increase of this class of lipids; moreover, when water stress conditions became more severe, a PG decrease and a pronounced increase of typical lipid classes of green tissues (MGDG and DGDG) were observed as well as ALA (18:3n-3) in PC lipid class and DGDG [48]. Likewise, it was reported the same PG increase in Pachyrhizus ahipa leaves [49]; furthermore, in barley roots grown in the presence of $100 \mathrm{mM} \mathrm{NaCl}, \mathrm{PG}$

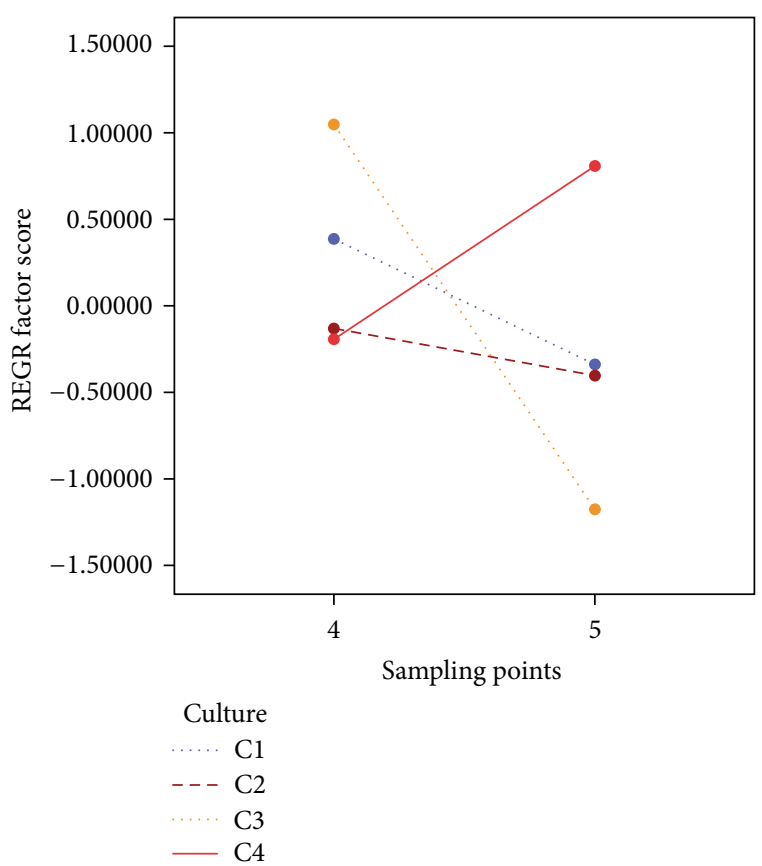

FIGURE 4: REGR factor score for PC1 depending on sampling point 4 or 5 and categorized by type of culture (C1-C4), showing the interaction effect of abiotic stress and time.

reached around 5\% of TL [65] and, in Carthamus tinctorius leaves, where PG and the set of polar lipids especially PC rose under smooth water stress conditions but decreased significantly when water conditions became more acute [55]. On the other hand, PG content was not influenced by time or sampling point or by the interaction of both in the culture (Table 4).

Therefore, when samples were plotted as a function of the main components and stratified according to the presence of stressing factors, a clear separation between the control group, culture $\mathrm{C} 1$, or the other cultures (C2-C4), also grown at $15^{\circ} \mathrm{C}$, was observed (Figure $5(\mathrm{a})$ ). When these samples were stratified according to the sampling points, an overlap between the groups was observed (Figure 5(b)).

3.3. Effect of Stress on the Unsaturation Degree of the Fatty Acids. The FA detected in E. acanthocarpum hairy roots were the same as those reported previously [36], but the percentages of each FA changed considerably. Thus, the following saturated FA were detected: palmitic acid (16:0) and stearic acid (18:0), along with other minor FA like 20:0, 22:0, and 24:0 (Tables 5 and 6). Similarly, high percentages of the monounsaturated FA $18: \ln -9$ and $18: \ln -7$ were measured, with a total of approximately $28 \%$ of saturated FA, with $6 \%$ of monoenes in all samples. The more characteristic polyunsaturated n-6 FA, LA and GLA, represented 50-55\% of the total FA. Furthermore, the n-3 PUFA, ALA and SDA, represented 6-9\% of all FA, depending on the cultures (Tables 5 and 6). 


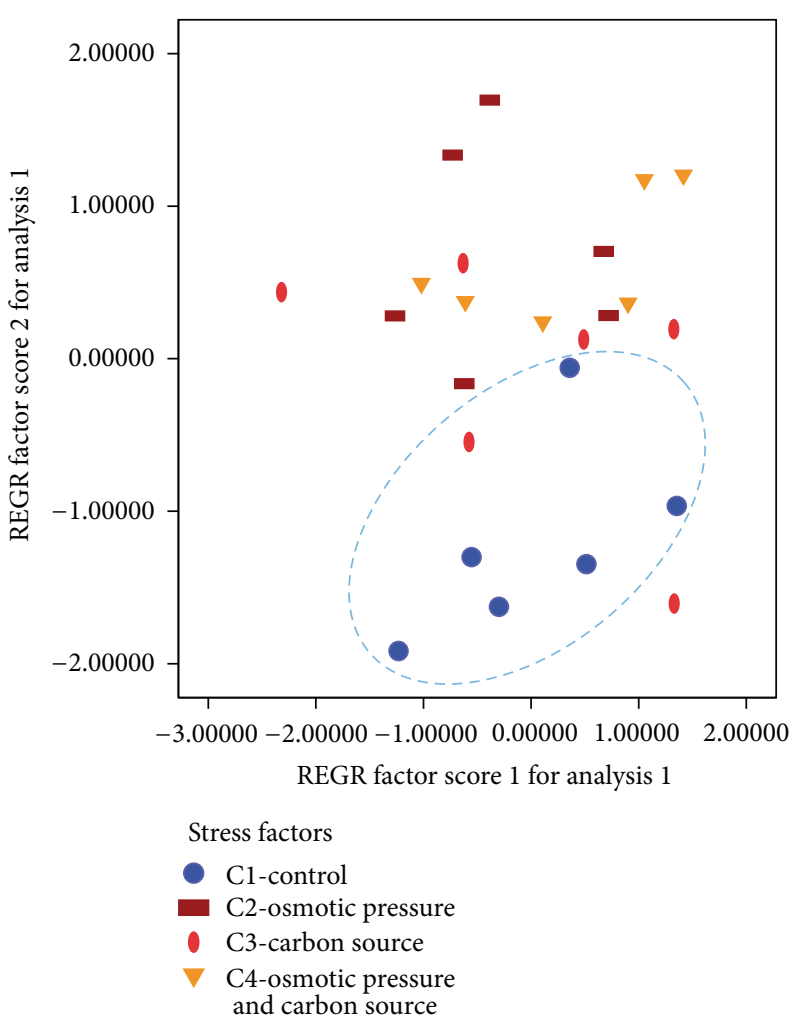

(a)

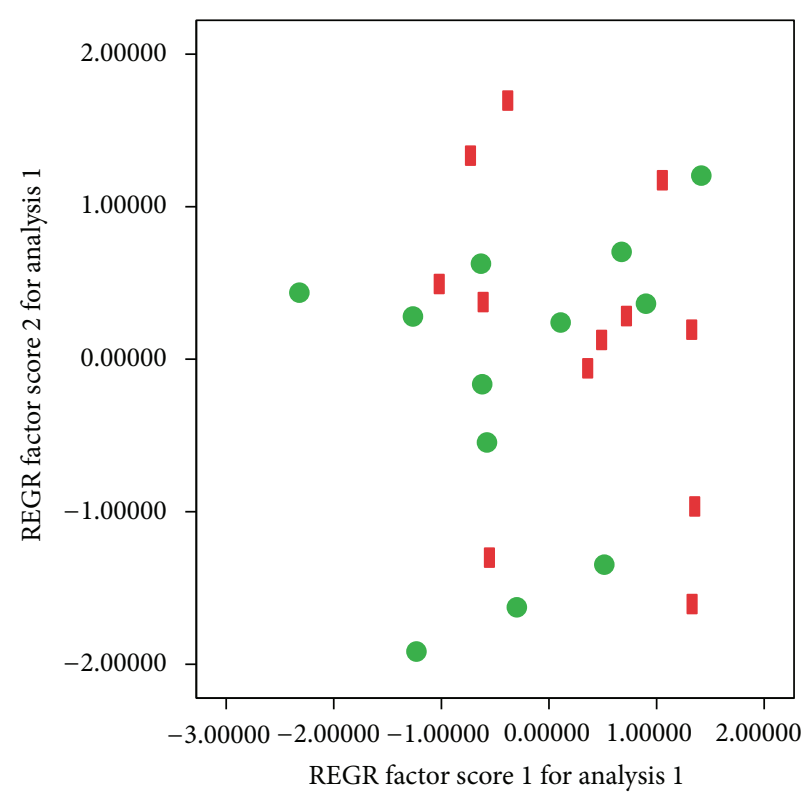

Sampling points

4

5

Figure 5: (a), (b) Plot of Echium acanthocarpum hairy root samples in terms of the principal components PC1 and PC2 stratified according to (a) cultures (C1-C4) or (b) sampling points.

TABLE 3: Principal components (PC1, PC2) of the studied lipid classes in Echium acanthocarpum E1.5 cell line hairy roots, growing in different culture media at $15^{\circ} \mathrm{C}(\mathrm{C} 1-\mathrm{C} 4)$. Factor loadings and communalities are shown.

\begin{tabular}{lccc}
\hline Components matrix & Components & & $\begin{array}{c}\text { Communalities } \\
\text { Extraction }\end{array}$ \\
\hline PC & PC1 (65.91\%) & PC2 (19.78\%) & 0.900 \\
PS + PI & $\mathbf{0 . 8 8 6}$ & -0.339 & 0.585 \\
PG & $\mathbf{0 . 7 5 9}$ & 0.094 & 0.934 \\
PE & 0.033 & 0.966 & 0.735 \\
Polar lipid & $\mathbf{0 . 7 6 7}$ & 0.382 & 0.975 \\
Neutral lipid & $\mathbf{0 . 8 4 6}$ & 0.510 & 0.850 \\
\hline
\end{tabular}

Factor loading of the variable (correlations) with PC1 are shown in bold, and those correlated with PC2 are shown in italic.

PC: phosphatidylcholine; PS + PI: phosphatidylserine and phosphatidylinositol; PG: phosphatidylglycerol; PE: phosphatidylethanolamine.

Rotation method: Varimax with Kaiser.

TABLE 4: Two-way ANOVA (stress conditions and sampling time) of the two principal components PC1 and PC2 of recorded lipid classes in Echium acanthocarpum E1.5 cell line hairy root cultures (C1-C4), growing at $15^{\circ} \mathrm{C}$.

\begin{tabular}{lccccccc}
\hline \multirow{2}{*}{ Two-way ANOVA } & \multicolumn{2}{c}{ Stress conditions } & \multicolumn{2}{c}{ Time } & \multicolumn{3}{c}{ Interaction between factors } \\
& (osmotic pressure and carbon source) & (sampling point) & \multicolumn{2}{c}{ Sign. } \\
\hline PC1 & F-value & Sign. & F-value & Sign. & F-value & 3.474 & 0.041 \\
PC2 & 0.167 & 0.917 & 3.379 & 0.085 & 0.197 & 2.434 & 0.103 \\
\hline
\end{tabular}

Sign.: significance $(P \leq 0.05)$. 
TABLE 5: Total lipid content (TL; mg/g DW), total fatty acid content (FA, mg/g DW), and the percentage of each fatty acid (\%) of Echium acanthocarpum hairy roots at sampling point 4 cultured for 20 days (culture B1) or 45 days (cultures C1-C4).

\begin{tabular}{|c|c|c|c|c|c|}
\hline & \multicolumn{5}{|c|}{ Sampling point 4} \\
\hline & B1 & $\mathrm{C} 1$ & $\mathrm{C} 2$ & $\mathrm{C} 3$ & $\mathrm{C} 4$ \\
\hline TL (mg/g DW) & $33.41 \pm 3.66$ & $41.67 \pm 2.45$ & $35.05 \pm 4.50$ & $32.20 \pm 4.94$ & $31.03 \pm 1.03$ \\
\hline FA (mg/g DW) & $7.30 \pm 6.28$ & $18.90 \pm 3.85$ & $9.74 \pm 0.55$ & $12.80 \pm 4.00$ & $8.92 \pm 1.26$ \\
\hline \multicolumn{6}{|l|}{ Fatty acids } \\
\hline $14: 0$ & $0.22 \pm 0.02$ & $0.15 \pm 0.01$ & ND & $0.10 \pm 0.09$ & $0.12 \pm 0.11$ \\
\hline $16: 0$ & $26.53 \pm 1.73$ & $20.03 \pm 0.99$ & $23.43 \pm 2.02$ & $23.02 \pm 2.40$ & $24.49 \pm 1.10$ \\
\hline 18:0 & $2.92 \pm 0.30$ & $1.48 \pm 0.06$ & $1.94 \pm 0.24$ & $1.88 \pm 0.30$ & $2.20 \pm 0.22$ \\
\hline $18: \ln -9$ & $4.56 \pm 0.92$ & $8.29 \pm 1.70$ & $5.69 \pm 0.71$ & $6.26 \pm 1.09$ & $4.94 \pm 1.07$ \\
\hline $18: \ln -7$ & $1.53 \pm 0.05$ & $0.60 \pm 0.04$ & $0.87 \pm 1.19$ & $0.90 \pm 0.16$ & $1.10 \pm 0.07$ \\
\hline $18: 2 n-6$ (LA) & $36.82 \pm 0.99$ & $35.79 \pm 0.65$ & $33.98 \pm 2.00$ & $35.71 \pm 1.09$ & $36.70 \pm 1.41$ \\
\hline 18:3n-6 (GLA) & $13.88 \pm 0.78$ & $18.37 \pm 0.70$ & $18.99 \pm 2.51$ & $18.15 \pm 1.51$ & $17.69 \pm 1.53$ \\
\hline 18:3n-3 (ALA) & $4.24 \pm 0.20$ & $5.16 \pm 0.65$ & $6.71 \pm 0.80$ & $6.17 \pm 0.56$ & $6.16 \pm 0.54$ \\
\hline $18: 4 n-3$ (SDA) & $1.26 \pm 0.45$ & $1.96 \pm 0.47$ & $3.06 \pm 0.67$ & $2.77 \pm 0.43$ & $1.29 \pm 0.89$ \\
\hline $20: 0$ & $0.29 \pm 0.06$ & $0.45 \pm 0.04$ & $0.60 \pm 0.03$ & $0.53 \pm 0.08$ & $0.57 \pm 0.10$ \\
\hline $22: 0$ & $2.45 \pm 0.31$ & $1.56 \pm 0.12$ & $1.71 \pm 0.16$ & $1.60 \pm 0.04$ & $1.55 \pm 0.18$ \\
\hline $24: 0$ & $1.94 \pm 0.49$ & $0.18 \pm 0.02$ & $1.28 \pm 0.07$ & $1.37 \pm 0.12$ & $0.29 \pm 0.06$ \\
\hline Unknown & $2.36 \pm 0.35$ & $2.63 \pm 0.71$ & $1.26 \pm 0.25$ & $1.25 \pm 0.32$ & $1.50 \pm 0.06$ \\
\hline GLA + SDA & $15.14 \pm 1.23$ & $20.33 \pm 1.17$ & $22.05 \pm 3.18$ & $20.92 \pm 1.94$ & $18.98 \pm 2.42$ \\
\hline Total saturated FA & $34.36 \pm 1.67$ & $25.36 \pm 0.92$ & $28.97 \pm 2.09$ & $28.50 \pm 2.54$ & $30.33 \pm 1.08$ \\
\hline Total monoene FA & $7.07 \pm 0.73$ & $10.72 \pm 1.90$ & $7.03 \pm 0.70$ & $7.45 \pm 0.90$ & $6.33 \pm 1.15$ \\
\hline$n-9$ & $5.33 \pm 0.66$ & $9.94 \pm 1.95$ & $5.69 \pm 0.71$ & $6.26 \pm 1.09$ & $4.94 \pm 1.07$ \\
\hline$n-6$ & $50.70 \pm 1.66$ & $54.16 \pm 0.95$ & $52.97 \pm 0.54$ & $53.85 \pm 1.51$ & $54.39 \pm 2.87$ \\
\hline$n-3$ & $5.50 \pm 0.53$ & $7.12 \pm 1.10$ & $9.76 \pm 1.46$ & $8.94 \pm 0.94$ & $7.45 \pm 0.84$ \\
\hline$n-3 / n-6$ & $0.11 \pm 0.01$ & $0.13 \pm 0.02$ & $0.18 \pm 0.03$ & $0.17 \pm 0.02$ & $0.14 \pm 0.02$ \\
\hline$\Delta 6$-des index $(n-6)$ & $0.27 \pm 0.01$ & $0.34 \pm 0.01$ & $0.36 \pm 0.04$ & $0.34 \pm 0.02$ & $0.32 \pm 0.01$ \\
\hline$\Delta 6$-des index $(n-3)$ & $0.23 \pm 0.06$ & $0.27 \pm 0.02$ & $0.31 \pm 0.02$ & $0.31 \pm 0.02$ & $0.17 \pm 0.10$ \\
\hline DBI & $1.40 \pm 0.06$ & $1.61 \pm 0.03$ & $1.64 \pm 0.09$ & $1.63 \pm 0.07$ & $1.56 \pm 0.03$ \\
\hline
\end{tabular}

ND: Not detected. n-6 and n-3 $\Delta 6$-desaturation indexes were calculated as 18:3n-6/(18:3n-6+18:2n-6) and 18:4n-3/(18:4n-3+18:3n-3), respectively. Double bond index $(\mathrm{DBI})$ was calculated as $[(\% 18: 1 \mathrm{n})+2 \times(\% 18: 2 \mathrm{n})+3 \times(\% 18: 3 \mathrm{n})+4 \times(18: 4 \mathrm{n})] / 100$. Values represent the mean of three replicates $(n=3) \pm$ SD.

Again, sampling points 4 and 5 were the most representative, according to the biomass reached and the FA profiles displayed $[36,38]$.

3.3.1. Fatty Acid Profile of Culture C1 of Echium acanthocarpum Hairy Roots. Analyses and comparison of the data of FA profiles and the different indeces were performed after one-way ANOVA test. The FA profile appeared very homogeneous even after 55 days of culture (Table 6, Supplementary Tables S1-S4 in Supplementary Material available online at http://dx.doi.org/10.5402/2013/169510 additional information). There were only a few statistical differences along the different sampling points, mainly regarding the percentages of minor saturated FA as well as SDA. The 14:0 was significantly more abundant at sample point 1 ( $0.26 \%$ of total FA), while 18:0 was more abundant at sampling points 1 and 2 ( $2.61 \%$ and 1.83 of total FA). However, values for 24:0 were significantly higher at the end of the culture recording $2.70 \%$ of total FA (Tables S1-S4 additional information).

Regarding the main PUFA present in the samples, that is, LA (18:2n-6) and GLA (18:3n-6), no significant differences were observed (Tables 5 and 6; S1-S4 additional information). LA values were slightly higher at sampling point 1 , with a total of $38.11 \%$ of the total FA, although this difference was not significant. Regarding the other sampling points, LA values ranged from 34 to $36 \%$. The n-6 $\Delta 6$-desaturated FA, GLA, reached slightly higher values in the middle of the culture (17.51 to $19.23 \%$ ), corresponding to the exponential phase of the growth curve, although these differences were not statistically significant (Tables 5 and 6; S1-S4 additional information). With regard to the n-3 PUFA, ALA showed 5-6\% of total FA, almost constant over time (Tables 5 and 6; S1-S4 additional information). On the other hand, SDA showed 1.62 to $2.12 \%$ of total FA, being its percentages significantly higher along sampling points 3-6 (Tables 5 and 6; S1-S4 additional information). It is well known that biological membranes adjust their composition according to the environmental conditions [66, 67], and they are also the most susceptible part of the cell to temperature decline, requiring to adjust their fluidity and permeability to maintain membrane functionality [68], which is achieved basically by increasing FA unsaturation degree $[11,13,45,69-72]$ and in 
TABLE 6: Total lipid content (TL; mg/g DW), total fatty acids content (FA; mg/g DW), and percentages of each fatty acid of Echium acanthocarpum hairy roots at sampling point 5 cultured for 25 days (culture B1) or 55 days (cultures C1-C4).

\begin{tabular}{|c|c|c|c|c|c|}
\hline & \multicolumn{5}{|c|}{ Sampling point 5} \\
\hline & B1 & $\mathrm{C} 1$ & $\mathrm{C} 2$ & $\mathrm{C} 3$ & $\mathrm{C} 4$ \\
\hline TL (mg/g DW) & $35.00 \pm 5.40$ & $35.58 \pm 7.33$ & $28.59 \pm 6.17$ & $42.96 \pm 5.50$ & $28.34 \pm 4.17$ \\
\hline FA (mg/g DW) & $8.45 \pm 0.87$ & $13.71 \pm 4.10$ & $9.38 \pm 0.84$ & $13.29 \pm 3.43$ & $9.90 \pm 1.71$ \\
\hline \multicolumn{6}{|l|}{ Fatty acids } \\
\hline $14: 0$ & $0.23 \pm 0.02$ & $0.14 \pm 0.03$ & $0.06 \pm 0.10$ & $0.08 \pm 0.14$ & $0.11 \pm 0.09$ \\
\hline $16: 0$ & $25.04 \pm 1.14$ & $20.21 \pm 1.81$ & $22.02 \pm 0.33$ & $21.66 \pm 0.21$ & $23.38 \pm 0.70$ \\
\hline 18:0 & $2.55 \pm 0.21$ & $1.82 \pm 0.48$ & $1.91 \pm 0.24$ & $1.93 \pm 0.31$ & $1.86 \pm 0.15$ \\
\hline $18: \ln -9$ & $6.77 \pm 1.10$ & $8.79 \pm 2.43$ & $4.82 \pm 1.14$ & $5.66 \pm 0.49$ & $4.24 \pm 1.65$ \\
\hline $18: \ln -7$ & $1.55 \pm 0.16$ & $0.70 \pm 0.06$ & $0.74 \pm 0.09$ & $0.86 \pm 0.26$ & $0.97 \pm 0.14$ \\
\hline $18: 2 n-6$ (LA) & $34.55 \pm 1.97$ & $34.34 \pm 3.77$ & $34.14 \pm 3.44$ & $31.95 \pm 2.95$ & $38.08 \pm 1.47$ \\
\hline 18:3n-6 (GLA) & $11.77 \pm 1.63$ & $17.51 \pm 1.77$ & $19.79 \pm 0.61$ & $19.92 \pm 0.98$ & $19.23 \pm 0.27$ \\
\hline 18:3n-3 (ALA) & $4.22 \pm 0.34$ & $5.32 \pm 0.56$ & $5.85 \pm 1.60$ & $5.96 \pm 1.17$ & $6.22 \pm 0.40$ \\
\hline $18: 4 n-3$ (SDA) & $0.88 \pm 0.21$ & $2.12 \pm 0.19$ & $2.73 \pm 0.47$ & $2.75 \pm 0.43$ & $1.27 \pm 0.55$ \\
\hline $20: 0$ & $0.27 \pm 0.04$ & $0.64 \pm 0.30$ & $0.84 \pm 0.25$ & $0.80 \pm 0.38$ & $0.42 \pm 0.05$ \\
\hline $22: 0$ & $2.51 \pm 0.35$ & $2.42 \pm 1.50$ & $2.52 \pm 1.84$ & $2.83 \pm 1.32$ & $1.35 \pm 0.09$ \\
\hline $24: 0$ & $2.08 \pm 0.60$ & $0.21 \pm 0.02$ & $0.40 \pm 0.17$ & $1.81 \pm 0.45$ & $0.21 \pm 0.03$ \\
\hline Unknown & $6.08 \pm 2.68$ & $2.23 \pm 0.46$ & $1.60 \pm 1.12$ & $3.50 \pm 1.64$ & $1.23 \pm 0.53$ \\
\hline $\mathrm{GLA}+\mathrm{SDA}$ & $12.65 \pm 1.84$ & $19.63 \pm 1.96$ & $22.52 \pm 1.08$ & $22.67 \pm 1.43$ & $20.50 \pm 0.82$ \\
\hline Total saturated FA & $32.68 \pm 2.21$ & $27.15 \pm 4.25$ & $29.37 \pm 2.84$ & $29.11 \pm 2.58$ & $28.55 \pm 0.60$ \\
\hline Total monoene FA & $9.54 \pm 1.29$ & $11.32 \pm 2.42$ & $6.52 \pm 1.91$ & $6.81 \pm 0.61$ & $5.42 \pm 1.81$ \\
\hline$n-9$ & $7.59 \pm 1.15$ & $10.40 \pm 2.41$ & $5.38 \pm 2.04$ & $5.66 \pm 0.49$ & $4.24 \pm 1.65$ \\
\hline$n-6$ & $46.32 \pm 3.60$ & $51.85 \pm 2.00$ & $53.93 \pm 3.67$ & $51.87 \pm 2.31$ & $57.31 \pm 1.70$ \\
\hline$n-3$ & $5.09 \pm 0.53$ & $7.44 \pm 0.42$ & $8.58 \pm 2.07$ & $8.71 \pm 1.54$ & $7.49 \pm 0.16$ \\
\hline$n-3 / n-6$ & $0.11 \pm 0.01$ & $0.14 \pm 0.01$ & $0.16 \pm 0.03$ & $0.17 \pm 0.00$ & $0.13 \pm 0.00$ \\
\hline$\Delta 6$-des index (n-6) & $0.25 \pm 0.02$ & $0.34 \pm 0.05$ & $0.37 \pm 0.02$ & $0.38 \pm 0.02$ & $0.34 \pm 0.01$ \\
\hline$\Delta 6$-des index $(n-3)$ & $0.17 \pm 0.02$ & $0.29 \pm 0.04$ & $0.32 \pm 0.02$ & $0.32 \pm 0.03$ & $0.17 \pm 0.07$ \\
\hline DBI & $1.30 \pm 0.08$ & $1.57 \pm 0.05$ & $1.63 \pm 0.12$ & $1.59 \pm 0.10$ & $1.63 \pm 0.03$ \\
\hline
\end{tabular}

n- 6 and n-3 $\Delta 6$-desaturation indexes were calculated as 18:3n-6/(18:3n-6+18:2n-6) and 18:4n-3/(18:4n-3+18:3n-3), respectively. Double Bond Index (DBI) was calculated as $[(\% 18: \ln )+2 \times(\% 18: 2 \mathrm{n})+3 \times(\% 18: 3 \mathrm{n})+4 \times(18: 4 \mathrm{n})] / 100$. Values represent the mean of three replicates $(n=3) \pm$ SD.

particular augmenting those FA with three unsaturations, reporting a rise in 18:3n-3 (ALA), at the expense of declining 18:2n-6 (LA), depending on plant species characteristics [21, $45,61,71,73-78]$. In the case of E. acanthocarpum hairy roots, the rise of unsaturations was mainly due to the increase of $\Delta 6$-desaturated FA, in particular GLA, also supported by the general richness in double bonds of the FA samples as shown by the double bond index (DBI) data, which was clearly augmented when reducing the culture temperature from 25 to $15^{\circ} \mathrm{C}$ (Figure 6), similar to other reports $[45,49,72,75,79]$.

Although very few statistical differences were recorded for the FA contents, surprisingly, the analysis of the different calculated indexes and ratios showed significant differences for most of them along the different sampling points. The (n-6) $\Delta 6$-desaturation index showed slightly higher values of approximately 0.34 , at sampling points 4 and 5 (Tables 5 and 6), parallel to higher values observed for GLA, which were approximately $17-18 \%$ of total FA. Likewise, significant differences were also found for the other $\Delta 6$-desaturation index, (n-3), which was maximum, 0.27 to 0.29 , also at sampling points 4 and 5 and paralleled with changes in SDA (Tables 5 and 6). In addition, the evolution of the DBI was studied, whose values were significantly higher among sampling points $2-5$ (1.55 to 1.65) (Figure 6, Tables 5 and 6; S1-S4 additional information).

When the FA contents were expressed in absolute values, it was observed for the hairy roots grown at $15^{\circ} \mathrm{C}$ (Figure 7) that LA was the most abundant FA, $6,473.82 \mu \mathrm{g} / \mathrm{gDW}$ at sampling point 4 , representing a 2.15 -fold increase compared to culture $\mathrm{B} 1$ grown at $25^{\circ} \mathrm{C}$, with a minimum value of $4,007.68 \mu \mathrm{g} / \mathrm{gDW}$ at sampling point 1 . GLA was also very abundant, 3,316.92 $\mu \mathrm{g} / \mathrm{gDW}$ also at sampling point 4 , a 3.31-fold increase compared to culture $\mathrm{B} 1$ grown at $25^{\circ} \mathrm{C}$, with a minimum of $1,594.88 \mu \mathrm{g} / \mathrm{gDW}$ at sampling point 1 . More importantly, the FA of the n-3 series, ALA reached $1,046.32 \mu \mathrm{g} / \mathrm{gDW}$, representing a 2.75-fold increase compared to culture B1 grown at $25^{\circ} \mathrm{C}$ and SDA $391.07 \mu \mathrm{g} / \mathrm{gDW}$, a remarkable 3 -fold increase compared to culture $\mathrm{B} 1$ grown at $25^{\circ} \mathrm{C}$, both at sampling point 4 (Figures 7 and 8). Due to their importance and abundance in the sample, values of 16:0 and 18:1n-9 FA were also interesting. The maximum amounts of 16:0 (4,031 $\mathrm{gg} / \mathrm{gDW})$ and 18:1n-9 (1,715 $\mu \mathrm{g} / \mathrm{gDW})$ were recorded at sampling point 4 , with lower values at other sampling points (Figure 7). 


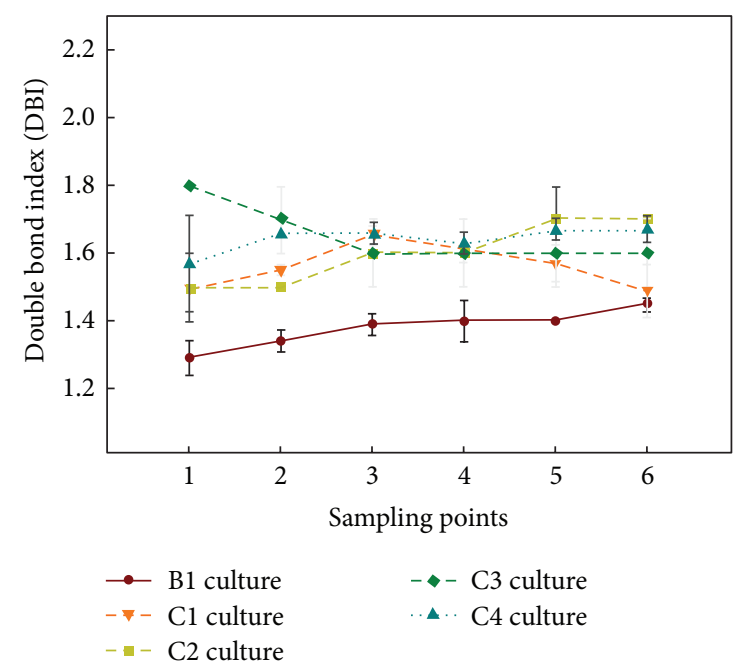

Figure 6: Double bond index (DBI) in Echium acanthocarpum hairy roots cultured in different growth media at two temperatures. DBI was calculated as $[(\% 18: 1 n)+2 \times(\% 18: 2 n)+3 \times(\% 18: 3 n)+4 \times$ $(18: 4 n)] / 100$.

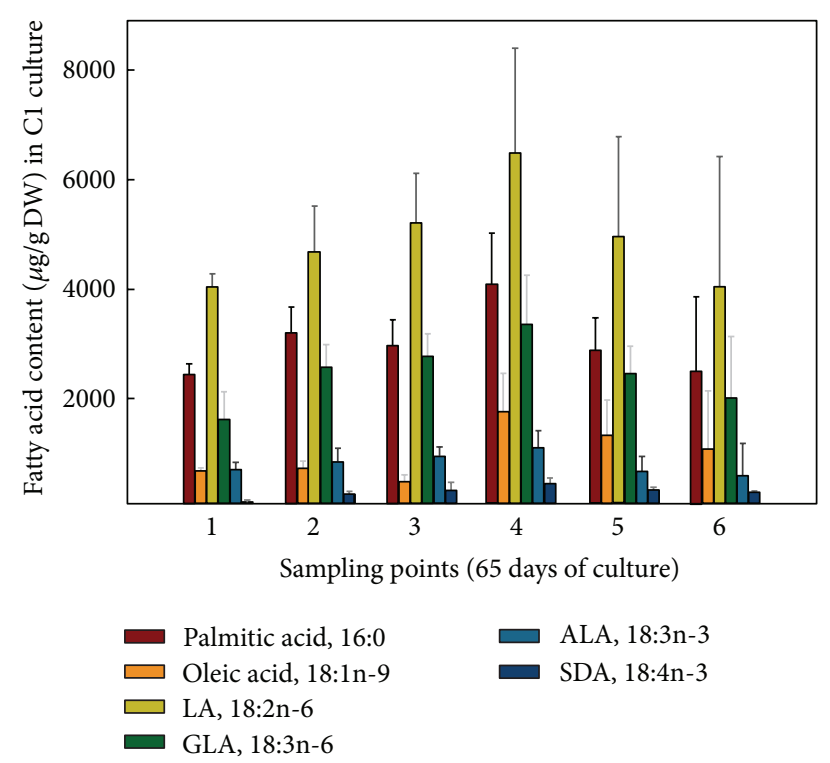

Figure 7: Absolute amounts of different fatty acids ( $\mu \mathrm{g} / \mathrm{gDW}$ ) present in Echium acanthocarpum hairy roots grown in B5 medium, $3 \%$ sucrose, $1 \% \mathrm{PVP}$ at $15^{\circ} \mathrm{C}$ (culture $\mathrm{C} 1$ ). Each value is the mean \pm standard deviation of three replicates.

As quoted above, these values were much higher than those achieved by the same hairy roots but grown at $25^{\circ} \mathrm{C}$ (culture B1, control) (Figure 8), whose maximum values were 3,000 and $1,000 \mu \mathrm{g} / \mathrm{gDW}$ for LA and GLA, respectively; moreover, FA of the n-3 series were recorded in lower quantities; ALA $380 \mu \mathrm{g} / \mathrm{gDW}$ (sampling point 5) and SDA $130 \mu \mathrm{g} / \mathrm{gDW}$ (sampling point 1) (Figure 6).

Another possible explanation to the increase in PUFA would be given precisely by the direct influence of temperature decrease on the expression of different desaturase coding

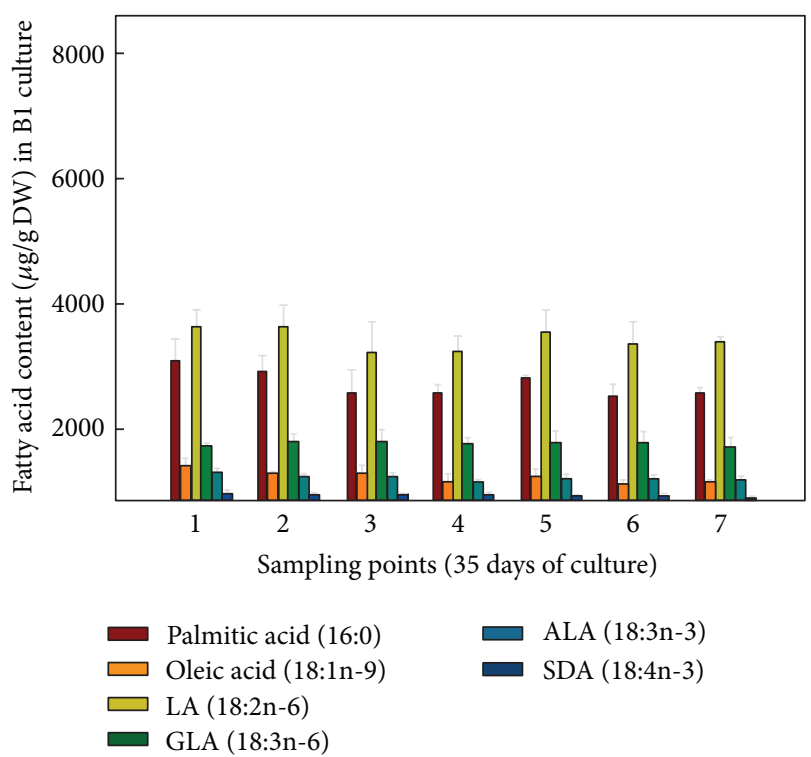

Figure 8: Absolute amounts of different fatty acids ( $\mu \mathrm{g} / \mathrm{gDW})$ present in Echium acanthocarpum hairy roots grown in B5 medium, $3 \%$ sucrose, $1 \% \mathrm{PVP}$ at $25^{\circ} \mathrm{C}$ (control culture $\mathrm{B} 1$ ). Each value is the mean \pm standard deviation of three replicates.

genes, transcriptional, translational or posttranscriptionaly. This fact was observed for the first time in soybean [22] and later in cyanobacterium [80], followed by others reports [7, 23, 66, 81-83]. Moreover, in rice, a different regulation at the transcriptional level of two desaturases (FAD7, FAD8) depending on culture temperature was reported $[84,85]$. This regulation is so effective that Arabidopsis mutants that lacked the FAD5 desaturase and being deficient in 16:1 desaturated products showed the same FA profiles compared with wild plants at the same temperature, with the levels of 18:2n-6 remaining constant, demonstrating the importance of this FA in plasma membrane homeostasis [70]. In E. acanthocarpum hairy roots, values of $\Delta 6$-desaturation index were higher when culture temperature decreased, suggesting that gene expression and/or the $\Delta 6$-desaturase activity itself may be temperature dependent although other influences may also take place.

3.3.2. Fatty Acid Profile of Culture C2 of Echium acanthocarpum Hairy Roots. Analogously, the same parameters and ratios were evaluated when hairy roots were grown in culture $\mathrm{C} 2$, with the addition of $0.2 \mathrm{M}$ sorbitol, as osmotic agent, and also at a temperature of $15^{\circ} \mathrm{C}$. FA profiles vaguely differed from those observed for culture $\mathrm{Cl}$, although significant differences along the sampling points were found for minor FA, such as stearic acid (18:0), which showed maximum values at sampling points 1 and 2 (Tables S1-S2 additional information), with percentages of 2.62 to 3.11 of total FA. There were also statistically significant differences for $18: 1 n$ 7, exhibiting also higher values in the first three sampling points, with percentages of 1.01 to 1.33 . (Tables S1-S3 additional information). Furthermore, the more unsaturated FA studied, that is, GLA and SDA, showed lower percentages at 
sampling point $1,12.72$ and 0.77 , respectively, with maximum values of 3\% for SDA and 19\% for GLA at sampling points 3-6 (Tables 5 and 6). In regard to LA and ALA, their values were relatively constant throughout the culture, with values of 33.98 to $39.10 \%$ for LA and 5.85 to $7.33 \%$ for ALA.

The n-6 $\Delta 6$-desaturation index showed significant differences, with a slightly lower value (0.25) at sampling point 1 , corresponding to the lowest value of GLA (12.72\%) (Table S1 additional information). Contrarily, the highest values were observed at sampling points 4-5 (Tables 5 and 6), ranging from 0.36 to 0.37 , respectively, although they were not statistically different from the other values. By contrast, the n-3 $\Delta 6$-desaturation index showed significant differences, with maximum values of 0.29 to 0.32 , at sampling points $4-$ 6 (Tables 5 and 6; S3 additional information); again, these data paralleled the SDA variations. Finally, the DBI showed no significant differences, with values being higher at later culture time, coinciding with the stationary phase (1.63 to 1.69) (Figure 6).

In terms of absolute values, the highest amounts of both $\Delta 6$-desaturated FA, GLA and SDA, 1,880 and $303 \mu \mathrm{g} / \mathrm{gDW}$, respectively, were obtained at sampling point 4 (Figure 9), being slightly lower than those obtained in nonsorbitol containing culture $\mathrm{C} 1$ (Figure 7). Again, LA was the most abundant FA, displaying $4,114 \mu \mathrm{g} / \mathrm{gDW}$ at sampling point 1 . Besides, the maximum value of ALA was $771 \mu \mathrm{g} / \mathrm{gDW}$ at sampling point 2, which was also lower than that recorded in culture $\mathrm{C} 1$ (Figures 7 and 9).

Therefore, comparing the results obtained with culture $\mathrm{C} 1$ (at $15^{\circ} \mathrm{C}$ ) with those of culture $\mathrm{C} 2$ with the addition of sorbitol (osmotic stress) also grown at $15^{\circ} \mathrm{C}$, the combination of these two factors did not further increase the yield of the important $\Delta 6$-desaturated FA under investigation, that is, ALA, SDA, LA, and GLA; moreover, the lower amounts of FA recorded in culture $\mathrm{C} 2$ well correlate to the lower percentage of total FA in the lipid extract (Tables 5 and 6).

\subsubsection{Fatty Acid Profile of Culture C3 of Echium acantho-} carpum Hairy Roots. When hairy roots were grown in the presence of $3 \%$ glucose instead of $3 \%$ sucrose also at $15^{\circ} \mathrm{C}$, similar FA values were observed; although, in general, significant differences were less frequent. Contrarily to the yield in culture $\mathrm{C} 2$, here SDA was significantly more abundant at sampling point 1 , reaching an attractive $3.45 \%$ of total FA (Table S1, additional information), which was statistically similar to those recorded at sampling points 3-6, ranging from 2.37 to $2.77 \%$ of total FA (Tables 5 and 6 ; S2, S4 additional information). For the rest of PUFA present in the samples, the percentage values were statistically similar throughout time. LA exhibited total amounts of 31.95 to $35.93 \%$ of total FA, while ALA reached amounts from 5.87 to $8.30 \%$, with GLA showing similar values to those achieved by the other cultures, from 17.58 to $20.13 \%$ of total FA (Tables 5 and 6).

In this culture, the ratio $n-3 / n-6$ showed a significantly higher value $(0.21)$ at sampling point 1 , due to the increased in SDA at the same data point, which was $3.45 \%$ (Table S1 additional information). For the remaining data points, the

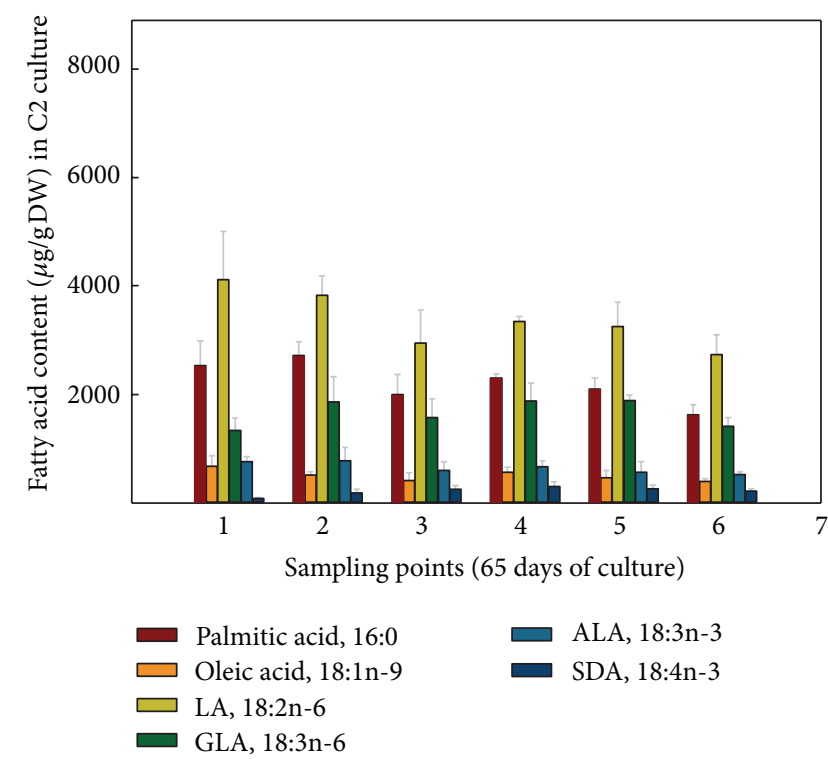

Figure 9: Absolute amounts of different fatty acids ( $\mu \mathrm{g} / \mathrm{gDW}$ ) present in Echium acanthocarpum hairy roots grown in B5 medium, $3 \%$ sucrose, $0.2 \mathrm{M}$ sorbitol, $1 \% \mathrm{PVP}$, at $15^{\circ} \mathrm{C}$ (culture $\mathrm{C} 2$ ). Each value is the mean \pm standard deviation of three replicates expressed.

value of this index was 0.17 , except at sampling point $6(0.15)$ (Tables 5 and 6; S2-S4 additional information). It was also observed that the value for n-3 $\Delta 6$-desaturation index was significantly lower (0.22) at sampling point 2 but ranging from 0.29 to 0.32 at other sampling points (Tables 5 and 6; S2S4 additional information). Likewise, significant differences on DBI were observed with a minimum value of 1.55 again at sampling point 2 but between 1.59 and 1.75 for the other time points (Figure 6).

Interestingly, FA values expressed in $\mu \mathrm{g} / \mathrm{gDW}$ showed maximum PUFA amounts at sampling point 1 (Figure 10), due in part to the greater amount of TL extracted at this point (Figure 2). Furthermore, at sampling points 3-5, PUFA amounts remained very stable, LA, 4,449-4,997; ALA 809885; GLA, 2,388-2,724; and SDA of 369-389 $\mu \mathrm{g} / \mathrm{gDW}$; that is, values are slightly lower than those obtained for cultures C1C2.

3.3.4. Fatty Acid Profile of Culture C4 of Echium acanthocarpum Hairy Roots. Finally, hairy roots were grown in a medium containing $0.2 \mathrm{M}$ sorbitol and $3 \%$ glucose and also at $15^{\circ} \mathrm{C}$. In general, the same trends in terms of percentages recorded for each FA were observed. Significant differences were detected for some saturated FA with palmitic acid (16:0) and stearic acid (18:0) showing lower values at the last sampling point, with $21.66 \%$ and $1.81 \%$ of total FA, respectively (Tables 5 and 6; S4 additional information). The major PUFA showed no significant differences, with LA being slightly more abundant than for the other cultures $(\mathrm{C} 1-\mathrm{C} 3)$ grown at $15^{\circ} \mathrm{C}$, with values ranging from $36.70 \%$ to $38.08 \%$ of total FA (Tables 5 and 6; S1-S4 additional information). ALA, however, appeared to maintain the same percentages as for the other cultures, representing $6.16 \%$ to $7.75 \%$ of 

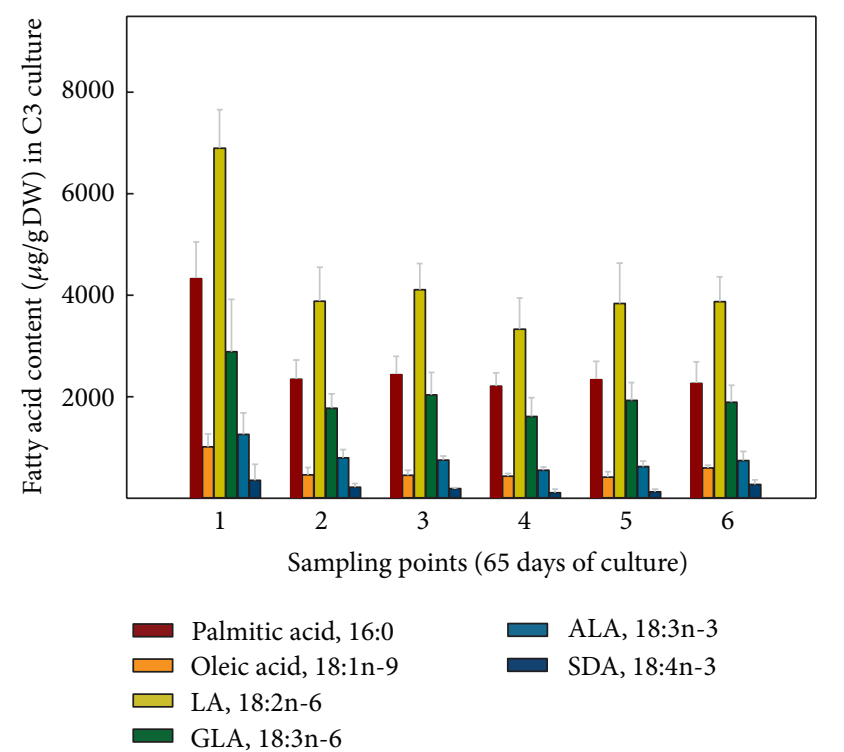

Figure 10: Absolute amounts of different fatty acids ( $\mu$ g/gDW) present in Echium acanthocarpum hairy roots grown in B5 medium, $3 \%$ glucose, $1 \% \mathrm{PVP}$ at $15^{\circ} \mathrm{C}$ (culture C3). Each value is the mean \pm standard deviation of three replicates.

total FA. The most unsaturated PUFA in the samples, GLA showed interesting percentages at sampling points 4 and 5 , with values between 18 and 19\% of total FA (Tables 5 and 6), while SDA, similar to $\mathrm{C} 3$ culture, which differs from $\mathrm{C} 4$ on the absence of osmotic pressure, showed higher values at the beginning of the culture, up to $2 \%$ of total FA and at the end of culture, where it reached $2.53 \%$ of total FA (Tables 5 and 6 ; S1-S4 additional information).

The different ratios and indexes studied did not show any statistical differences, with a maximum value of 0.34 (Table 6) for $\mathrm{n}-6 \Delta 6$-desaturation index at sampling point 5 and 0.21 and 0.26 at sampling points 2 and 6 , respectively, for the n-3 $\Delta 6$-desaturation index (Tables 6; S1-S4 additional information). The $n-3 / n-6$ ratio showed values of 0.13 to 0.18 throughout the culture, and DBI index showed 1.56 to 1.67 (Figure 6).

The absolute amounts of each FA displayed maximum values of PUFA at the first sampling point (Figure 11), corresponding also to the highest TL content (Figure 2). Meanwhile, for the remaining sampling points, TL was lower, and therefore the amounts of each FA also decreased, showing the following values: LA 3,335-4,112; ALA 555-795; GLA 1,614-2,036; and for SDA 112-215 $\mu \mathrm{g} / \mathrm{g}$ DW (Figure 11). These values were slightly lower than those recorded for cultures $\mathrm{C} 1$ and $\mathrm{C} 3$ but higher than those exhibited in culture C2. Moreover, here in culture C4, lower TL values were recorded, being significantly different compared to the other cultures at sampling point 4 (Figure 2), which would justify the lower FA amounts present in this culture parallel to the lower TL content, especially when compared with culture $\mathrm{Cl}$.

The influence of culture temperature $\left(25\right.$ versus $\left.15^{\circ} \mathrm{C}\right)$ on the unsaturation degree in the pool of FA was studied by principal component analyses, which allow to reduction of

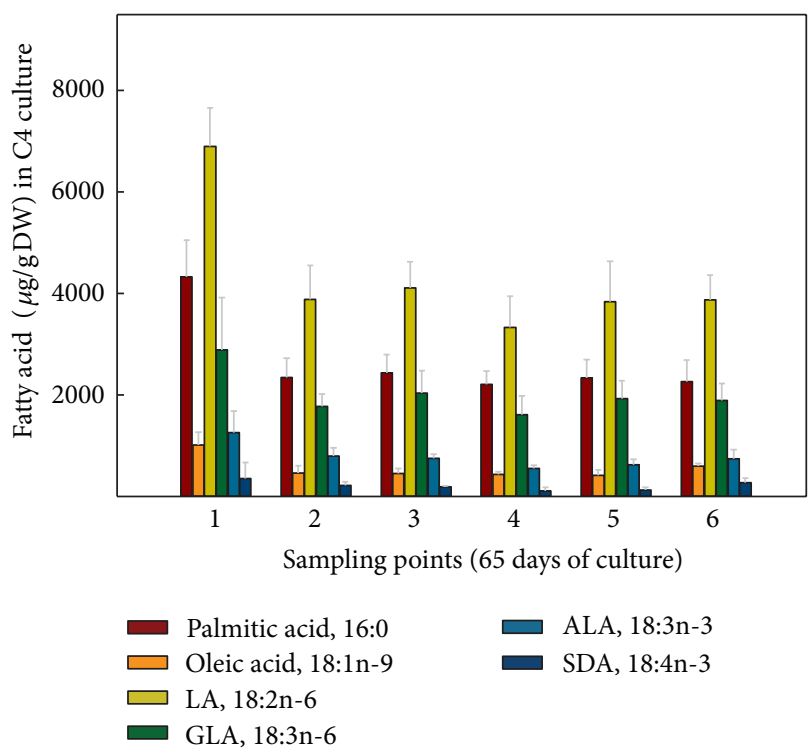

FIGURE 11: Absolute amounts of different fatty acids ( $\mu \mathrm{g} / \mathrm{gDW}$ ) present in Echium acanthocarpum hairy roots grown in B5 medium, $3 \%$ glucose, $0.2 \mathrm{M}$ sorbitol, $1 \% \mathrm{PVP}$ at $15^{\circ} \mathrm{C}$ (culture $\mathrm{C} 4$ ). Each value is the mean \pm standard deviation of three replicates.

the dimensionality of the variables. The statistical variables (12 different FA) were introduced for the cultures B1 and $\mathrm{Cl}$, since the only difference between these cultures was the temperature at which hairy roots had been grown, $25^{\circ} \mathrm{C}$ and $15^{\circ} \mathrm{C}$, respectively, with culture $\mathrm{B} 1$ selected as control. Two principal components ( $\mathrm{PCl}$ and $\mathrm{PC} 2$ ) were extracted, which explained $67.88 \%$ of the variance and were able to summarize the information contained in the 12 variables (FA). PCl, $48.95 \%$ of the variance, was positively correlated with the minor saturated FA 14:0, 18:0, and 24:0, with 16:0 and the monoene 18:1n-7 (Table 7, Figure 12). In addition, PC1 was also found to be correlated, although negatively, with the determinant FA involved in the PUFA metabolic pathway, that is, oleic acid (18:1n-9) and the $\Delta 6$-desaturated acids, GLA and SDA (Figure 12; Table 7). Moreover, PC2, 17.92\% of variance, was negatively correlated with the precursors of these FA, that is, LA and ALA, and positively correlated with 22:0 (Figure 12; Table 7).

Accordingly, it could be said that PC1 is again correlated with the abundance of saturated FA and inversely proportional to the adaptation of hairy roots to temperature, meaning that higher $\mathrm{PCl}$ values suggest a decrease in GLA and SDA and a lower relative activity of the $\triangle 6$-desaturase enzyme. On the other hand, and also inversely, PC2 reflects PUFA contents which are the substrates of the $\Delta 6$-desaturase, that is, LA and ALA. The same correlations were established when performing a principal component analysis to the absolute values of FA.

In order to test whether the studied factors, temperature and time of culture, and the interaction between them, would affect the obtained variables, $\mathrm{PC} 1$ and $\mathrm{PC} 2$, a twoway ANOVA test was performed. As shown in Table 8, the level of significance indicated that both temperature and 
TABLE 7: Principal components (PC1 y PC2) of fatty acids studied in Echium acanthocarpum hairy roots, growing at different temperatures $\left(25\right.$ and $\left.15^{\circ} \mathrm{C}\right)$ in culture media (B1, C1).

\begin{tabular}{lccc}
\hline Matrix components & Components & & $\begin{array}{c}\text { Communalities } \\
\text { Extraction }\end{array}$ \\
\hline $14: 0$ & PC1 (48.95\%) & PC2 (17.92\%) & 0.658 \\
$16: 0$ & $\mathbf{0 . 7 6 7}$ & 0.265 & 0.876 \\
$18: 0$ & $\mathbf{0 . 9 2 9}$ & 0.112 & 0.829 \\
$18: 1 \mathrm{n}-9$ & $\mathbf{0 . 8 9 6}$ & 0.163 & 0.317 \\
$18: 1 \mathrm{n}-7$ & $\mathbf{- 0 . 5 5 0}$ & 0.119 & 0.909 \\
$18: 2 \mathrm{n}-6$ & $\mathbf{0 . 9 4 9}$ & -0.091 & 0.710 \\
$18: 3 \mathrm{n}-6$ & 0.170 & -0.826 & 0.788 \\
$18: 3 \mathrm{n}-3$ & $-\mathbf{0 . 8 8 8}$ & -0.021 & 0.394 \\
$18: 4 \mathrm{n}-3$ & -0.335 & -0.530 & 0.509 \\
$20: 0$ & $-\mathbf{0 . 5 6 1}$ & 0.441 & 0.752 \\
$22: 0$ & $\mathbf{- 0 . 6 5 8}$ & 0.565 & 0.591 \\
$24: 0$ & 0.240 & 0.730 & 0.813 \\
\hline
\end{tabular}

bold values show factor loadings of variables (correlations) with PC1 and in italic the correlations with PC2.

Rotation method: Varimax with Kaiser normalization.

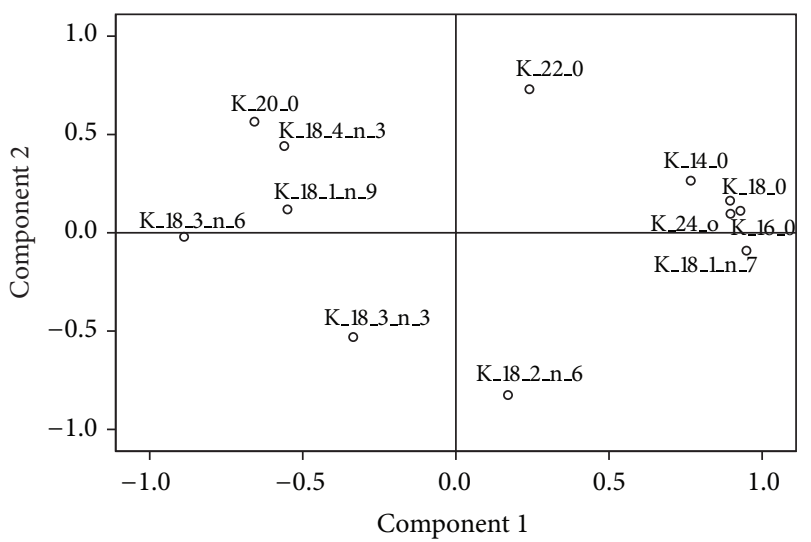

FIGURE 12: Factor loadings for the percentages of fatty acids in Echium acanthocarpum hairy root cultures $\mathrm{B} 1$ and $\mathrm{C} 1$ grown at $25^{\circ} \mathrm{C}$ and $15^{\circ} \mathrm{C}$, obtained after principal component analysis.

time had influenced PC1, while PC2 was only influenced by the interaction of both factors. Furthermore, when plotted, the samples were clearly separated according to the main components and stratified with the temperature (Figure S1(a) additional information). In contrast, when the same stratified representation was made regarding sampling time, no effect was observed and the groups clearly overlapped (Figure S1(b) additional information). However, the influence of time on $\mathrm{PC} 1$ when plotted against sampling points was strongly attenuated between sampling points 3 and 5 (Figure S2 additional information).

Following the establishment of a direct relationship between the decrease of culture temperature and the enrichment of PUFA in the sample, the possible influence of the carbon source and sorbitol on the FA was also analyzed. A new principal component analysis was performed, and different variables (FA) for cultures $\mathrm{C} 1, \mathrm{C} 2, \mathrm{C} 3$, and $\mathrm{C} 4$, grown at $15^{\circ} \mathrm{C}$ in the presence of osmotic stress (cultures $\mathrm{C} 2$ and $\mathrm{C} 4$ ) and $3 \%$ glucose as carbon source (C3 and C4), were analysed, choosing culture $\mathrm{Cl}$ as a control which also grew at $15^{\circ} \mathrm{C}$ but without sorbitol or glucose.

The principal components analysis of the percentages of FA identified two new components PC1 and PC2, which explain $52.50 \%$ of the data (Figure 13, Table 9).

PC1 (30.77\% of variance) was positively correlated with the saturated FA, 16:0 and 18:0, and with the monoene $18: 1 n-7$. Furthermore, $\mathrm{PCl}$ was negatively correlated with the more unsaturated FA and products of the $\Delta 6$-desaturase enzyme activity, GLA and SDA. Whereas PC2 (21.73\% of the variance) was strongly and negatively correlated with LA and ALA, both $\Delta 6$-desaturase enzyme substrates, and was also associated with some minor saturated FA, 14:0, 20:0, 22:0, and 24:0. In other words, high values of the new PC2 variable suggest lower amounts of the $\Delta 6$-desaturase enzyme substrates (LA and GLA) and, possibly, an increase of the FA product of this enzyme (GLA and SDA). The PCA performed with the absolute amounts of FA also showed the same correlations.

A two-way ANOVA test showed a significant influence of both the stressing factors present in the cultures and factor time on $\mathrm{PCl}$ and $\mathrm{PC} 2$ as well as the interaction between the two on PCl (Table 10). When plotting the data of the samples based on PCl and PC2 and stratifying them according to the stressing factors (different cultures), a separation between the groups was observed, especially for those samples belonging to culture C4 (Figure S3 additional information), in which osmotic stress was applied by the presence of sorbitol. In contrast, when plotting the data stratified according to sampling points, a greater overlap between the groups was also observed (data not shown).

Post-hoc analysis performed for each of the principal components revealed that cultures pairs $\mathrm{C} 1-\mathrm{C} 2$ and $\mathrm{C} 3-\mathrm{C} 4$ were statistically equal for PC1 (Figures S4(a)-4(d) additional information). The lowest $\mathrm{PCl}$ values corresponded with 
TABLE 8: Two-way ANOVA test (stress factor and time) of PC1 and PC2 of detected fatty acids from Echium acanthocarpum hairy roots, grown at different temperature $25^{\circ} \mathrm{C}$ and $15^{\circ} \mathrm{C}$ (cultures $\mathrm{B} 1, \mathrm{C} 1$ ).

\begin{tabular}{lcccccc}
\hline \multirow{2}{*}{ Two-way ANOVA } & \multicolumn{2}{c}{ Stress factor (Temperature) } & \multicolumn{2}{c}{ Time (Sampling point) } & \multicolumn{3}{c}{ Interaction between factors } \\
& $F$-value & Sign. & $F$-value & Sign. & $F$-value & Sign. \\
\hline PC1 & 214.056 & 0.000 & 7.734 & 0.000 & 2.174 & 0.091 \\
PC2 & 1.126 & 0.299 & 0.464 & 0.799 & 3.162 & 0.025 \\
\hline
\end{tabular}

PC: principal component; Sign.: significance $(P \leq 0.05)$.

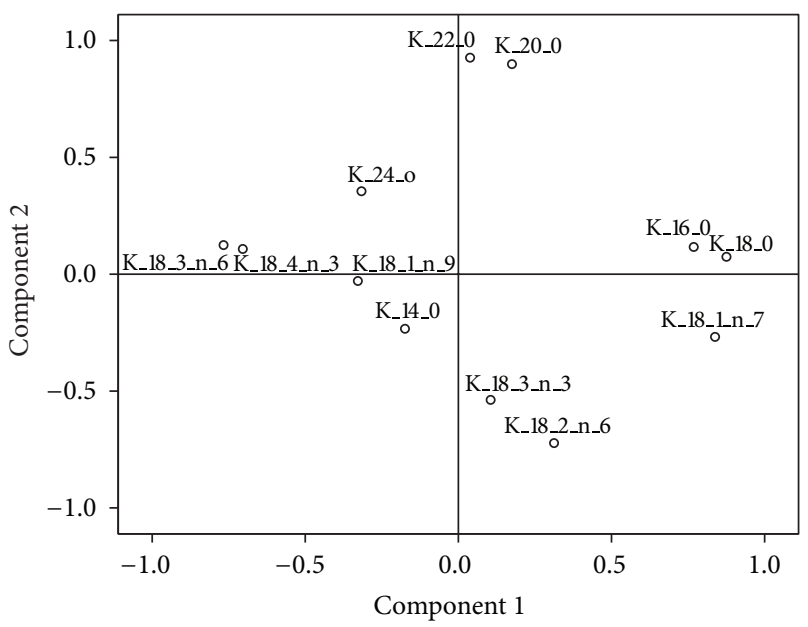

FIGURE 13: Factor loadings obtained after principal component analysis, for the percentages of fatty acids in Echium acanthocarpum hairy root cultures $\mathrm{C} 1-\mathrm{C} 4$ grown at $15^{\circ} \mathrm{C}$.

culture $\mathrm{C} 1$ (control) and culture $\mathrm{C} 3$, in which $3 \%$ glucose was added as carbon source, indicating that these cultures showed, in general, less content of saturated FA, a higher content of the PUFA, GLA and SDA, and a relatively higher $\Delta 6$-desaturase activity.

Moreover, for $\mathrm{PCl}$, the values were significantly higher at sampling points 1 and 2 (Figure S4(b) additional information), and points 3-5 appeared more favorable in order to obtain a more abundant set of PUFA. The PC2 post-hoc study displayed significant differences in culture C4 (Figures S3, S4(c) additional information). Considering that PC2 was a reflex of the low content of the $\Delta 6$-desaturase substrates, LA and ALA, culture C4 would be the one containing the largest amounts of these two FA. This theoretically suggests a lower $\Delta 6$-desaturase activity and, therefore, fewer amounts of the PUFA, GLA and SDA, being in full agreement with the results observed for PC1. Regarding the influence of the sampling points on PC2, a parity between the sampling points 1-4 and 2-6 was observed, with sampling points 4-5 appearing as the most appropriate since these showed a high percentage of the target PUFA of this study, GLA and SDA (Figure S4(d) additional information), and the tendency which suggests that sampling points 4 and 5 would be the most appropriate because of the high TL content (Figure 2).

Under water stress conditions, which is mimicked in in vitro cultures by applying osmotic stress, reports do not show an overall increase of unsaturation, even more a decrease of 18:3n-3 was found, the most unsaturated FA present in the plant species studied which was a reflex of the damage caused by stress $[48,49,86]$. When water stress conditions become more severe, this FA percentage can be increased, as in the experiments conducted in the leaves of A. thaliana [48].

In contrast, in other cases, the use of osmotic stress or high osmotic pressure to stimulate the production of secondary metabolites in plant cell cultures appeared as an effective strategy [25-31]. A possible explanation of this fact could be that stress conditions inhibit initially cell growth and division and, hence, increase the concentration of cell culture carbohydrates, thus being a more available resources for secondary metabolism [30]. However, in E. acanthocarpum hairy roots, no significant increase or decrease in fresh weight of cultures C2 and C4 was registered, which may correlates with this hypothesis (Figure 1).

In ginseng roots, the added sorbitol may act by activating the phenylalanine amino-lyase enzyme, increasing the production of reactive oxygen species (ROS). ROS are also closely related to the response of plants to low temperatures [11]; however, the presence of ROS was not studied in our research.

In E. acanthocarpum hairy root system, the combined possible effects produced at low temperature and osmotic stress do not seem to be cumulative on the FA profile. Regarding the influence of the carbon source on the FA profile, it is necessary to note that, in plants FA synthesis occur predominantly in plastids and require a carbon source, ATP, and reducing power. The carbon source for the FA synthesis is usually in the form of acetyl-coenzyme A (acetylCoA), which is not able to cross the plastid membrane. Thus, precursors of acetyl-CoA should be generated inside the plastids or be imported from the cytosol. In heterotrophic seeds, lipids may accumulate in large quantities, for example, comprising up to $40 \%$ dry weight of the embryo, as in sunflower seeds, forcing a massive influx of carbon source into the plastids to maintain synthesis [87]. Thus, various studies have shown that a wide range of cytosolic metabolites, such as glucose-6-P, phosphoenolpyruvate (PEP) and pyruvate malate, are capable of withstanding the FA biosynthesis [9]. Moreover, the ATP required for the synthesis of FA in nonphotosynthetic tissues, such as E. acanthocarpum hairy roots, occurs in the plastids, from the synthesis of acetyl-CoA, glucose-6P, and PEP $[88,89]$. Despite this fact, in vitro studies of plastids, isolated from sunflower seeds, reported that malate was the carbon source supporting a better FA biosynthesis, although these results were not in 
TABLE 9: Principal components (PC1 y PC2) of fatty acid profiles studied in Echium acanthocarpum hairy roots, growing in different culture media (C1-C4).

\begin{tabular}{lccc}
\hline Matrix Components & Components & Communalities & Extraction \\
& PC1 $(30.772 \%)$ & PC2 $(21.729 \%)$ & 0.085 \\
$14: 0$ & -0.174 & -0.233 & 0.604 \\
$16: 0$ & $\mathbf{0 . 7 6 9}$ & 0.116 & 0.772 \\
$18: 0$ & $\mathbf{0 . 8 7 6}$ & 0.074 & 0.109 \\
$18: 1 \mathrm{n}-9$ & -0.328 & -0.029 & 0.775 \\
$18: 1 \mathrm{n}-7$ & $\mathbf{0 . 8 3 8}$ & -0.268 & 0.620 \\
$18: 2 \mathrm{n}-6$ & 0.313 & -0.723 & 0.604 \\
$18: 3 \mathrm{n}-6$ & $-\mathbf{0 . 7 6 7}$ & 0.125 & 0.300 \\
$18: 3 \mathrm{n}-3$ & 0.105 & -0.538 & 0.507 \\
$18: 4 \mathrm{n}-3$ & $-\mathbf{0 . 7 0 4}$ & 0.108 & 0.839 \\
$20: 0$ & 0.175 & 0.899 & 0.860 \\
$22: 0$ & 0.038 & 0.926 & 0.226 \\
$24: 0$ & -0.316 & 0.355 & \\
\hline
\end{tabular}

Bold values show factor loadings of variables (correlations) with PC1 and in italic the factor loadings the correlations with PC2.

Rotation method: Varimax with Kaiser normalization.

TABLE 10: Two-way ANOVA (stress conditions and sampling time) of PC1 and Trans-PC2 of fatty acid profiles in Echium acanthocarpum hairy roots, growing in different culture media at $15^{\circ} \mathrm{C}$ (cultures $\mathrm{C} 1-\mathrm{C} 4$ ).

\begin{tabular}{lcccccc}
\hline \multirow{2}{*}{ Two-way ANOVA } & \multicolumn{2}{c}{ Stress conditions } & \multicolumn{2}{c}{ Time } & \multicolumn{2}{c}{ Interaction between factors } \\
& (osmotic pressure and carbon source) & (sampling point) & \multicolumn{2}{c}{ Sign. } \\
\hline PC1 & $F$-value & Sign. & $F$-value & Sign. & 8.315 & 0.000 \\
Trans-PC2 & 13.912 & 0.000 & 24.725 & 0.000 & 1.610 & 0.106 \\
\hline
\end{tabular}

PC: principal component; Trans-PC2: transformed principal component 2 (NL (PC2-min (PC2) +1)); Sign.: significance $(P \leq 0.05)$.

agreement with those obtained in vivo, where malate was the minor source $[9,87]$. A more recent study, which used corn embryos, showed that the main carbon source for the synthesis of FA was PEP originated from hexose-P [90, 91]. These authors also suggested that the glucose dehydrogenase6-phosphate played an important role in the provision of the reducing power in the form of NADPH for FA biosynthesis in seeds, where photosynthesis is restricted.

\section{Conclusions}

The results presented here show the usefulness and high potential of the E. acanthocarpum hairy roots for biosynthesizing and accumulating a large range of polyunsaturated FA, including the target SDA and GLA in appreciable quantities; furthermore, from the point of view of production and accumulation of SDA and GLA, E. acanthocarpum hairy roots are more efficient and boosted PUFA yields when grown in a nutrient medium consisting of B5 basal salts, sucrose or glucose as a carbon source and more importantly at a temperature of at least $15^{\circ} \mathrm{C}$ and for a period of not less than 45-55 days (sampling points 4 and 5), resulting in a drop of LA but a strong rise of SDA (60\%) and more moderate for GLA (35\%). On the other hand, the application of osmotic stress did not seem to exert a positive influence on the amount of PUFA accumulated, and the combination of a lower culture temperature and glucose did not show a cumulative boosting effect on PUFA production; although this carbon source was similarly attractive proving the appropriateness of the strategy of applying abiotic stress in this novel culture system to enhance FA yields. Using this culture system, further research results are being evaluated on the effects of the overexpression of the $\Delta 6$-desaturase gene from Primula vialii, which specifically catalyzes the conversion of ALA into SDA, after applying also the abiotic stress conditions which yielded the highest results presented here.

\section{Conflict of Interests}

The authors declare no conflict of interests and do not have any financial relation with any of the commercial identities mentioned in the paper which have been provided for a better description of the methods employed.

\section{Authors' Contribution}

Rafael Zárate conceived the project, coordinated, and supervised it, designed the study, revised the data, and contrived and wrote the paper; Elena Cequier-Sánchez carried out the experiments, analyzed and processed the experimental data, and contributed to writing the paper. Nabil El Jaber-Vazdekis assisted in evaluating the data and writing the paper; Roberto Dorta-Guerra designed and supervised all statistical analyses of the data; Covadonga Rodríguez coordinated the project, 
revised the data, and refined the paper; Ángel G. Ravelo contributed towards lab infrastructure and assisted in the performance of some analytical techniques.

\section{Acknowledgments}

The authors acknowledge the funding from the Spanish Ministry of Science, Research and Innovation of Spain, and the Ministry of Economy and Competitiveness (Projects MTM2010-16828, SAF2012-37344-C03-01, AGL-200306877-C01-ACU, and CTM-2006-14279-CO2-02-MAR), and Agencia Canaria de Investigación, Innovación y Sociedad de la Información (ACIISI) \& FSE (Project CLU20101006) as well as Canary Islands Cancer Research Institute (ICIC). ECS also acknowledges a scholarship from "Programa de Formación de Profesorado Universitario-FPU” MEC, Spain.

\section{References}

[1] M. Orešič, V. A. Hänninen, and A. Vidal-Puig, "Lipidomics: a new window to biomedical frontiers," Trends in Biotechnology, vol. 26, no. 12, pp. 647-652, 2008.

[2] R. A. Siddiqui, K. Harvey, and W. Stillwell, "Anticancer properties of oxidation products of docosahexaenoic acid," Chemistry and Physics of Lipids, vol. 153, no. 1, pp. 47-56, 2008.

[3] P. C. Calder, "Omega-3 fatty acids and inflammatory processes," Nutrients, vol. 2, no. 3, pp. 355-374, 2010.

[4] D. F. Horrobin, "Nutritional and medical importance of gamma-linolenic acid," Progress in Lipid Research, vol. 31, no. 2, pp. 163-194, 1992.

[5] C. J. A. W. van Gool, C. Thijs, C. J. M. Henquet et al., "Gamma-linolenic acid supplementation for prophylaxis of atopic dermatitis-a randomized controlled trial in infants at high familial risk," The American Journal of Clinical Nutrition, vol. 77, no. 4, pp. 943-951, 2003.

[6] J. P. Schuchardt, M. Huss, M. Stauss-Grabo, and A. Hahn, "Significance of long-chain polyunsaturated fatty acids (PUFAs) for the development and behaviour of children," European Journal of Pediatrics, vol. 169, no. 2, pp. 149-164, 2010.

[7] M. O. Winfield, C. Lu, I. D. Wilson, J. A. Coghill, and K. J. Edwards, "Plant responses to cold: transcriptome analysis of wheat," Plant Biotechnology Journal, vol. 8, no. 7, pp. 749-771, 2010.

[8] O. L. Gamborg, R. A. Miller, and K. Ojima, "Nutrient requirements of suspension cultures of soybean root cells," Experimental Cell Research, vol. 50, no. 1, pp. 151-158, 1968.

[9] R. Pleite, M. J. Pike, R. Garcés, E. Martínez-Force, and S. Rawsthorne, "The sources of carbon and reducing power for fatty acid synthesis in the heterotrophic plastids of developing sunflower (Helianthus annuus L.) embryos," Journal of Experimental Botany, vol. 56, no. 415, pp. 1297-1303, 2005.

[10] C. D. Stubbs and A. D. Smith, "The modification of mammalian membrane polyunsaturated fatty acid composition in relation to membrane fluidity and function," Biochimica et Biophysica Acta, vol. 779, no. 1, pp. 89-137, 1984.

[11] K. Iba, "Acclimative response to temperature stress in higher plants: approaches of gene engineering for temperature tolerance," Annual Review of Plant Biology, vol. 53, pp. 225-245, 2002.

[12] Y. Murakami, M. Tsuyama, Y. Kobayashi, H. Kodama, and K. Iba, "Trienoic fatty acids and plant tolerance of high temperature," Science, vol. 287, no. 5452, pp. 476-479, 2000.
[13] W. E. Teague, N. L. Fuller, R. P. Rand, and K. Gawrisch, "Polyunsaturated lipids in membrane fusion events," Cellular \& Molecular Biology Letters, vol. 7, no. 2, pp. 262-264, 2002.

[14] B. Maresca and G. Kobayashi, "Changes in membrane fluidity modulate heat shock gene expression and produced attenuated strains in the dimorphic fungus Histoplasma capsulatum," Archives of Medical Research, vol. 24, no. 3, pp. 247-249, 1993.

[15] P. E. Tiku, A. Y. Gracey, A. I. Macartney, R. J. Beynon, and A. R. Cossins, "Cold-induced expression of $\Delta 9$-desaturase in carp by transcriptional and posttranslational mechanisms," Science, vol. 271, no. 5250, pp. 815-818, 1996.

[16] L. E. Cybulski, D. Albanesi, M. C. Mansilla, S. Altabe, P. S. Aguilar, and D. Mendoza, "Mechanism of membrane fluidity optimization: isothermal control of the Bacillus subtilis acyllipid desaturase," Molecular Microbiology, vol. 45, no. 5, pp. 1379-1388, 2002.

[17] D. Thewke, M. Kramer, and M. S. Sinensky, "Transcriptional homeostatic control of membrane lipid composition," Biochemical and Biophysical Research Communications, vol. 273, no. 1, pp. 1-4, 2000.

[18] A. Grover, M. Agarwal, S. Katiyar-Argawal, C. Sahi, and S. Argawal, "Production of high temperature tolerant transgenic plants through manipulation of membrane lipids," Current Science, vol. 79, no. 5, pp. 557-559, 2000.

[19] J. Cyril, G. L. Powell, R. R. Duncan, and W. V. Baird, "Changes in membrane polar lipid fatty acids of seashore paspalum in response to low temperature exposure," Crop Science, vol. 42, no. 6, pp. 2031-2037, 2002.

[20] J. G. Wallis and J. Browse, "Mutants of Arabidopsis reveal many roles for membrane lipids," Progress in Lipid Research, vol. 41, no. 3, pp. 254-278, 2002.

[21] J.-M. Routaboul, S. F. Fischer, and J. Browse, “Trienoic fatty acids are required to maintain chloroplast function at low temperatures," Plant Physiology, vol. 124, no. 4, pp. 1697-1705, 2000.

[22] T. M. Cheesbrought, "Changes in enzymes for fatty acid synthesis and desaturation during acclimation of developing soybean seeds to altered growth temperature," Plant Physiology, vol. 90, no. 2, pp. 760-764, 1989.

[23] J. M. Dyer, D. C. Chapital, J. W. Cary, and A. B. Pepperman, "Chilling-sensitive, post-transcriptional regulation of a plant fatty acid desaturase expressed in yeast," Biochemical and Biophysical Research Communications, vol. 282, no. 4, pp. 10191025, 2001.

[24] S. Ramachandra Rao and G. A. Ravishankar, "Plant cell cultures: chemical factories of secondary metabolites," Biotechnology Advances, vol. 20, no. 2, pp. 101-153, 2002.

[25] J. I. Smith, N. J. Smart, W. G. W. Kurz, and M. Misawa, "The use of organic and inorganic compounds to increase the accumulation of indole alkaloids in Catharanthus roseus (L.)," Journal of Experimental Botany, vol. 38, no. 9, pp. 1501-1506, 1987.

[26] C. B. Do and F. Cormier, "Accumulation of anthocyanins enhanced by a high osmotic potential in grape (Vitis vinifera $L$.) cell suspensions," Plant Cell Reports, vol. 9, no. 3, pp. 143-146, 1990.

[27] A. Tholakalabavi, J. J. Zwiazek, and T. A. Thorpe, "Effect of mannitol and glucose-induced osmotic stress on growth, water relations, and solute composition of cell suspension cultures of poplar (Populus deltoides var. Occidentalis) in relation to anthocyanin accumulation," In Vitro Cellular \& Developmental Biology, vol. 30, no. 3, pp. 164-170, 1994. 
[28] Y.-H. Zhang, J.-J. Zhong, and J.-T. Yu, "Effect of osmotic pressure on cell growth and production of ginseng saponin and poly saccharide in suspension cultures of Panax notoginseng," Biotechnology Letters, vol. 17, no. 12, pp. 1347-1350, 1995.

[29] G. C. Godoy-Hernández, F. A. Vázquez-Flota, and V. M. Loyola-Vargas, "The exposure to trans-cinnamic acid of osmotically stressed Catharanthus roseus cells cultured in a $1_{4^{-}}$ 1 bioreactor increases alkaloid accumulation," Biotechnology Letters, vol. 22, no. 11, pp. 921-925, 2000.

[30] S.-I. Kim, H.-K. Choi, J.-H. Kim, H.-S. Lee, and S.-S. Hong, "Effect of osmotic pressure on paclitaxel production in suspension cell cultures of Taxus chinensis," Enzyme and Microbial Technology, vol. 28, no. 2-3, pp. 202-209, 2001.

[31] J. Y. Wu, K. Wong, K. P. Ho, and L. G. Zhou, "Enhancement of saponin production in Panax ginseng cell culture by osmotic stress and nutrient feeding," Enzyme and Microbial Technology, vol. 36, no. 1, pp. 133-138, 2005.

[32] A. Ibarz, F. Padrós, M. Á. Gallardo, J. Fernández-Borràs, J. Blasco, and L. Tort, "Low-temperature challenges to gilthead sea bream culture: review of cold-induced alterations and 'Winter Syndrome," Reviews in Fish Biology and Fisheries, vol. 20, no. 4, pp. 539-556, 2010.

[33] A. R. Cossins and A. G. Macdonald, "Homeoviscous adaptation under pressure. III. The fatty acid composition of liver mitochondrial phospholipids of deep-sea fish," Biochimica et Biophysica Acta, vol. 860, no. 2, pp. 325-335, 1986.

[34] P. Sebert, "Fish at high pressure: a hundred year history," Comparative Biochemistry and Physiology A, vol. 131, no. 3, pp. 575-585, 2002.

[35] R. Zárate, N. El Jaber-Vazdekis, B. Medina, and Á. G. Ravelo, "Tailoring tropane alkaloid accumulation in transgenic hairy roots of Atropa baeticaby over-expressing the gene encoding hyoscyamine $6 \beta$-hydroxylase," Biotechnology Letters, vol. 28, no. 16, pp. 1271-1277, 2006.

[36] E. Cequier-Sánchez, C. Rodríguez, R. Dorta-Guerra, Á. G. Ravelo, and R. Zárate, "Echium acanthocarpum hairy root cultures, a suitable system for polyunsaturated fatty acid studies and production," BMC Biotechnology, vol. 11, article 42, 2011.

[37] J. Folch, M. Lee, and G. H. Sloane, "A simple method for the isolation and purification of total lipides from animal tissues," The Journal of Biological Chemistry, vol. 226, no. 1, pp. 497-509, 1957.

[38] E. Cequier-Sánchez, C. Rodríguez, Á. G. Ravelo, and R. Zárate, "Dichloromethane as a solvent for lipid extraction and assessment of lipid classes and fatty acids from samples of different natures," Journal of Agricultural and Food Chemistry, vol. 56, no. 12, pp. 4297-4303, 2008.

[39] W. W. Christie, "The isolation of lipid from tissues, the preparation of derivatives of lipids," in Lipid Analysis, W. W. Christie, Ed., chapter 2, pp. 17-23, 55-61, Pergamon Press Canada, Ontario, Canada, 1982.

[40] E. Cequier-Sánchez, Biotecnología del género Echium: aplicación para la producción de ácidos grasos de interés en medicina, acuicultura y nutrición [Ph.D. thesis], Univeristy of La Laguna, Tenerife, Spain, 2011.

[41] J. M. Al-Khayri and A. M. Al-Bahrany, "Callus growth and proline accumulation in response to sorbitol and sucroseinduced osmotic stress in rice," Biologia Plantarum, vol. 45, no. 4, pp. 609-611, 2002.

[42] M. Sajid Aqeel Ahmad, F. Javed, and M. Ashraf, "Iso-osmotic effect of $\mathrm{NaCl}$ and $\mathrm{PEG}$ on growth, cations and free proline accumulation in callus tissue of two indica rice (Oryza sativa L.) genotypes," Plant Growth Regulation, vol. 53, no. 1, pp. 5363, 2007.

[43] F. Javed and S. Ikram, "Effect of sucrose induced osmotic stress on callus growth and biochemical aspects of two wheat genotypes," Pakistan Journal of Botany, vol. 40, no. 4, pp. 14871495, 2008.

[44] J. Y. Wu, K. Wong, K. P. Ho, and L. G. Zhou, "Enhancement of saponin production in Panax ginseng cell culture by osmotic stress and nutrient feeding," Enzyme and Microbial Technology, vol. 36, no. 1, pp. 133-138, 2005.

[45] G. Tasseva, J. D. Virville, C. Cantrel, F. Moreau, and A. Zachowski, "Changes in the endoplasmic reticulum lipid properties in response to low temperature in Brassica napus," Plant Physiology and Biochemistry, vol. 42, no. 10, pp. 811-822, 2004.

[46] M. Miquel, D. James Jr., H. Dooner, and J. Browse, "Arabidopsis requires polyunsaturated lipids for low-temperature survival," Proceedings of the National Academy of Sciences of the United States of America, vol. 90, no. 13, pp. 6208-6212, 1993.

[47] A. Repellin, A. T. Pham, A. Tashakorie, Y. Sahsah, C. Daniel, and Y. Zuily-Fodil, "Leaf membrane lipids and drought tolerance in young coconut palms (Cocos nucifera L.)," European Journal of Agronomy, vol. 6, no. 1-2, pp. 25-33, 1997.

[48] A. Gigon, A.-R. Matos, D. Laffray, Y. Zuily-Fodil, and A.-T. Pham-Thi, "Effect of drought stress on lipid metabolism in the leaves of Arabidopsis thaliana (Ecotype Columbia)," Annals of Botany, vol. 94, no. 3, pp. 345-351, 2004.

[49] M. C. Matos, P. S. Campos, J. C. Ramalho et al., "Photosynthetic activity and cellular integrity of the Andean legume Pachyrhizus ahipa (Wedd.) Parodi under heat and water stress," Photosynthetica, vol. 40, no. 4, pp. 493-501, 2002.

[50] M. J. Utrillas and L. Alegre, "Impact of water stress on leaf anatomy and ultrastructure in Cynodon dactylon (L.) Pers. Under natural conditions," International Journal of Plant Sciences, vol. 158, no. 3, pp. 313-324, 1997.

[51] J. M. Farrant, K. Cooper, L. A. Kruger, and H. W. Sherwin, "The effect of drying rate on the survival of three desiccation-tolerant angiosperm species," Annals of Botany, vol. 84, no. 3, pp. 371379, 1999.

[52] J. Larkindale and M. R. Knight, "Protection against heat stressinduced oxidative damage in Arabidopsis involves calcium, abscisic acid, ethylene, and salicylic acid," Plant Physiology, vol. 128, no. 2, pp. 682-695, 2002.

[53] J. Larkindale and B. Huang, "Changes of lipid composition and saturation level in leaves and roots for heat-stressed and heat-acclimated creeping bentgrass (Agrostis stolonifera)," Environmental and Experimental Botany, vol. 51, no. 1, pp. 5767, 2004.

[54] K.-M. Oksman-Caldentey, N. Sevón, L. Vanhala, and R. Hiltunen, "Effect of nitrogen and sucrose on the primary and secondary metabolism of transformed root cultures of Hyoscyamus muticus," Plant Cell, Tissue and Organ Culture, vol. 38, no. 2-3, pp. 263-272, 1994.

[55] I. Hamrouni, H. B. Salah, and B. Marzouk, "Effects of waterdeficit on lipids of safflower aerial parts," Phytochemistry, vol. 58, no. 2, pp. 277-280, 2001.

[56] D. T. Clarkson, K. C. Hall, and J. K. M. Roberts, "Phospholipid composition and fatty acid desaturation in the roots of rye during acclimatization of low temperature," Planta, vol. 149, no. 5, pp. 464-471, 1980.

[57] G. Smolenska and P. J. C. Kuiper, "Effect of low temperature upon lipid and fatty acid composition of roots and leaves of 
winter rape plants," Physiologia Plantarum, vol. 41, no. 1, pp. 2935, 2006.

[58] H. A. Norman, C. Mcmillan, and G. A. Thompson, "Phosphatidylglycerol molecular species in chilling-sensitive and chilling-resistant populations of Avicennia germinans (L.)," Plant and Cell Physiology, vol. 25, no. 8, pp. 1437-1444, 1984.

[59] M. Bohn, S. Lüthje, P. Sperling, E. Heinz, and K. Dörffling, "Plasma membrane lipid alterations induced by cold acclimation and abscisic acid treatment of winter wheat seedlings differing in frost resistance," Journal of Plant Physiology, vol. 164, no. 2, pp. 146-156, 2007.

[60] N. Murata, O. Ishizaki-Nishizawa, S. Higashi, H. Hayashi, Y. Tasaka, and I. Nishida, "Genetically engineered alteration in the chilling sensitivity of plants," Nature, vol. 356, no. 6371, pp. 710713, 1992.

[61] Y.-N. Xu, Z.-N. Wang, G.-Z. Jiang, L.-B. Li, and T.-Y. Kuang, "Effect of various temperatures on phosphatidylglycerol biosynthesis in thylakoid membranes," Physiologia Plantarum, vol. 118, no. 1, pp. 57-63, 2003.

[62] M. Uemura, Y. Tominaga, C. Nakagawara, S. Shigematsu, A. Minami, and Y. Kawamura, "Responses of the plasma membrane to low temperatures," Physiologia Plantarum, vol. 126, no. 1, pp. 81-89, 2006.

[63] N. Murata, "Molecular species composition of phosphatidylglycerols from chilling-sensitive and chilling-resistant plants," Plant and Cell Physiology, vol. 24, no. 1, pp. 81-86, 1983.

[64] W. J. Wu Jingrui and J. Browse, "Elevated levels of high-meltingpoint phosphatidylglycerols do not induce chilling sensitivity in an Arabidopsis mutant," Plant Cell, vol. 7, no. 1, pp. 17-27, 1995.

[65] D. Brown and F. M. DuPont, "Lipid composition of plasma membranes and endomembranes prepared from roots of barley (Hordeum vulgareL.)," Plant Physiology, vol. 90, no. 3, pp. 955961, 1989.

[66] D. A. Los and N. Murata, "Membrane fluidity and its roles in the perception of environmental signals," Biochimica et Biophysica Acta, vol. 1666, no. 1-2, pp. 142-157, 2004.

[67] Y. Shi, L. An, M. Zhang, C. Huang, H. Zhang, and S. Xu, "Regulation of the plasma membrane during exposure to low temperatures in suspension-cultured cells from a cryophyte (Chorispora bungeana)," Protoplasma, vol. 232, no. 3-4, pp. 173181, 2008.

[68] L. Beney and P. Gervais, "Influence of the fluidity of the membrane on the response of microorganisms to environmental stresses," Applied Microbiology and Biotechnology, vol. 57, no. 12, pp. 34-42, 2001.

[69] H. Kodama, T. Hamada, G. Horiguchi, M. Nishimura, and K. Iba, "Genetic enhancement of cold tolerance by expression of a gene for chloroplast omega-3 fatty acid desaturase in transgenic tobacco," Plant Physiology, vol. 105, no. 2, pp. 601-605, 1994.

[70] D. L. Falcone, J. P. Ogas, and C. R. Somerville, "Regulation of membrane fatty acid composition by temperature in mutants of Arabidopsis with alterations in membrane lipid composition," BMC Plant Biology, vol. 4, article 17, pp. 1-45, 2004.

[71] F. Martz, S. Kiviniemi, T. E. Palva, and M.-L. Sutinen, "Contribution of omega-3 fatty acid desaturase and 3-ketoacyl-ACP synthase II (KASII) genes in the modulation of glycerolipid fatty acid composition during cold acclimation in birch leaves," Journal of Experimental Botany, vol. 57, no. 4, pp. 897-909, 2006.

[72] J. Bakht, A. Bano, and P. Dominy, "The role of abscisic acid and low temperature in chickpea (Cicer arietinum) cold tolerance. II. Effects on plasma membrane structure and function," Journal of Experimental Botany, vol. 57, no. 14, pp. 3707-3715, 2006.
[73] H. Kodama, G. Horiguchi, T. Nishiuchi, M. Nishimura, and K. Iba, "Fatty acid desaturation during chilling acclimation is one of the factors involved in conferring low-temperature tolerance to young tobacco leaves," Plant Physiology, vol. 107, no. 4, pp. 1177-1185, 1995.

[74] G. Horiguchi, T. Fuse, N. Kawakami, H. Kodama, and K. Iba, "Temperature-dependent translational regulation of the ER $\omega-3$ fatty acid desaturase gene in wheat root tips," The Plant Journal, vol. 24, no. 6, pp. 805-813, 2000.

[75] S.-Y. Chiou, W.-W. Su, and Y.-C. Su, "Optimizing production of polyunsaturated fatty acids in Marchantia polymorpha cell suspension culture," Journal of Biotechnology, vol. 85, no. 3, pp. 247-257, 2001.

[76] M. De Palma, S. Grillo, I. Massarelli et al., "Regulation of desaturase gene expression, changes in membrane lipid composition and freezing tolerance in potato plants," Molecular Breeding, vol. 21, no. 1, pp. 15-26, 2008.

[77] Z. Zhou, M.-J. Wang, J.-J. Hu, M.-Z. Lu, and J. H. Wang, "Improve freezing tolerance in transgenic poplar by overexpressing a $\omega-3$ fatty acid desaturase gene," Molecular Breeding, vol. 25, no. 4, pp. 571-579, 2010.

[78] R. P. da Cruz, J. I. Golombieski, M. T. Bazana, C. Cabreira, T. F. Silveira, and L. P. da Silva, "Alterations in fatty acid composition due to cold exposure at the vegetative stage in rice," Brazilian Journal of Plant Physiology, vol. 22, no. 3, pp. 199-207, 2010.

[79] D. L. Osmond, R. F. Wilson, and D. Raper, "Fatty acids composition and nitrate uptake of soybean roots during acclimatation to low temperature," Plant Physiology, vol. 70, no. 6, pp. 1689-1693, 1982.

[80] D. Los, I. Horvath, L. Vigh, and N. Murata, "The temperaturedependent expression of the desaturase gene desA in Synechocystis PCC6803," FEBS Letters, vol. 318, no. 1, pp. 57-60, 1993.

[81] S. Gibson, V. Arondel, K. Iba, and C. Somerville, "Cloning of a temperature-regulated gene encoding a chloroplast omega3 desaturase from Arabidopsis thaliana," Plant Physiology, vol. 106, no. 4, pp. 1615-1621, 1994.

[82] G.-Q. Tang, W. P. Novitzky, H. Carol Griffin, S. C. Huber, and R. E. Dewey, "Oleate desaturase enzymes of soybean: evidence of regulation through differential stability and phosphorylation," The Plant Journal, vol. 44, no. 3, pp. 433-446, 2005.

[83] G. E. Byfield and R. G. Upchurch, "Effect of temperature on delta-9 stearoyl-ACP and microsomal omega- 6 desaturase gene expression and fatty acid content in developing soybean seeds," Crop Science, vol. 47, no. 4, pp. 1698-1704, 2007.

[84] J. Wang, F. Ming, J. Pittman et al., "Characterization of a rice (Oryza sativa L.) gene encoding a temperature-dependent chloroplast $\omega$-3 fatty acid desaturase," Biochemical and Biophysical Research Communications, vol. 340, no. 4, pp. 1209-1216, 2006.

[85] P. M. Gopalakrishnan Nair, I.-S. Kang, B.-Y. Moon, and C.-H. Lee, "Effects of low temperature stress on rice (Oryza sativa L.) plastid $\omega$-3 desaturase gene, OsFAD8 and its functional analysis using T-DNA mutants," Plant Cell, Tissue and Organ Culture, vol. 98, no. 1, pp. 87-96, 2009.

[86] W. S. Dekhma, M. Zarrouk, and A. Cherif, "Effects of droughtstress on lipids in rape leaves," Phytochemistry, vol. 40, no. 5, pp. 1383-1386, 1995.

[87] A. P. Alonso, F. D. Goffman, J. B. Ohlrogge, and Y. ShacharHill, "Carbon conversion efficiency and central metabolic fluxes in developing sunflower (Helianthus annuus L.) embryos," The Plant Journal, vol. 52, no. 2, pp. 296-308, 2007. 
[88] Q. Q. Qi Qungang, K. F. Kleppinger-Sparace, and S. A. Sparace, "The role of the triose-phosphate shuttle and glycolytic intermediates in fatty-acid and glycerolipid biosynthesis in pea root plastids," Planta, vol. 194, no. 2, pp. 193-199, 1994.

[89] S. Rawsthorne, "Carbon flux and fatty acid synthesis in plants," Progress in Lipid Research, vol. 41, no. 2, pp. 182-196, 2002.

[90] R. J. Weselake, D. C. Taylor, M. H. Rahman et al., "Increasing the flow of carbon into seed oil," Biotechnology Advances, vol. 27, no. 6, pp. 866-878, 2009.

[91] A. Paula Alonso, V. L. Dale, and Y. Shachar-Hill, "Understanding fatty acid synthesis in developing maize embryos using metabolic flux analysis," Metabolic Engineering, vol. 12, no. 5, pp. 488-497, 2010. 

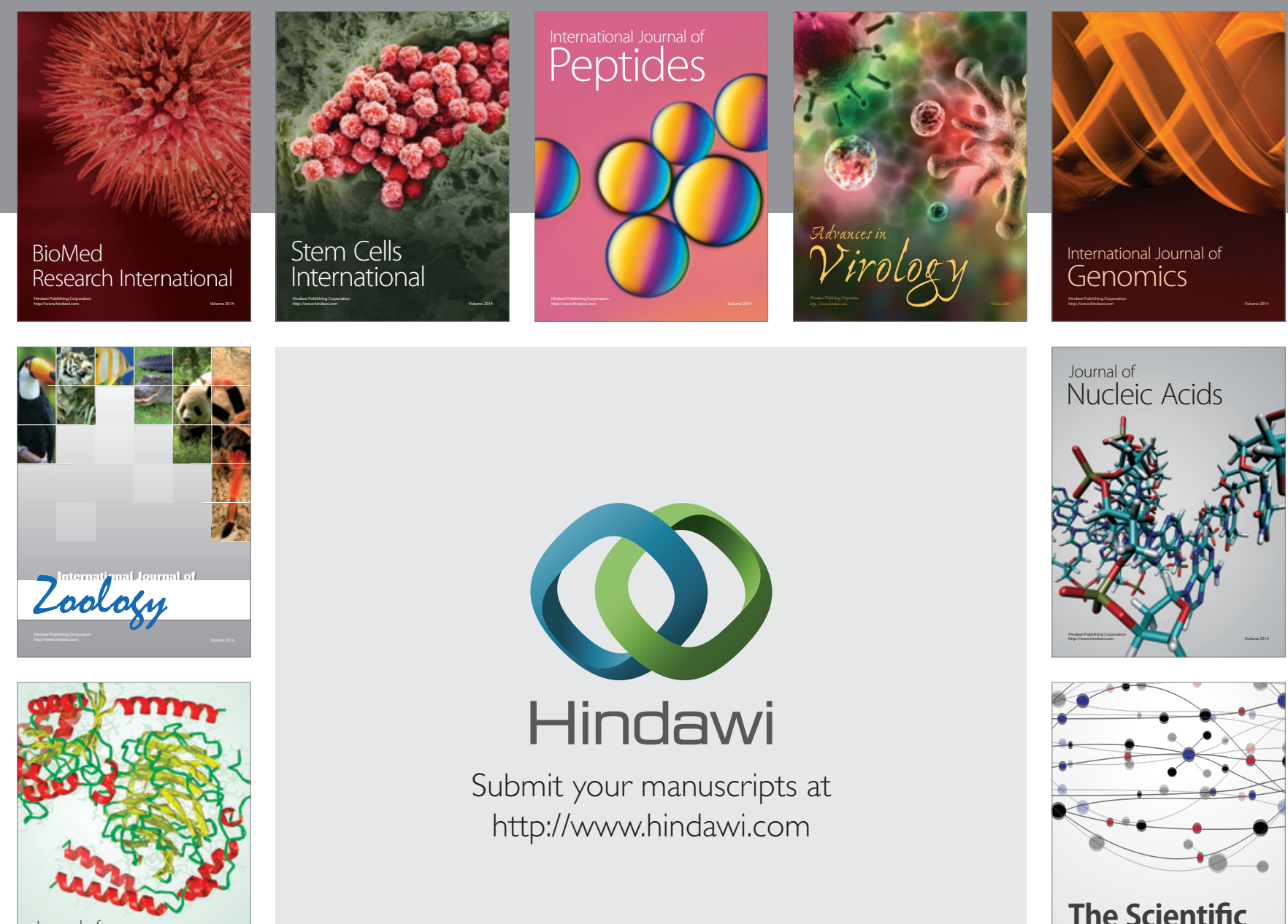

Submit your manuscripts at

http://www.hindawi.com

Journal of
Signal Transduction
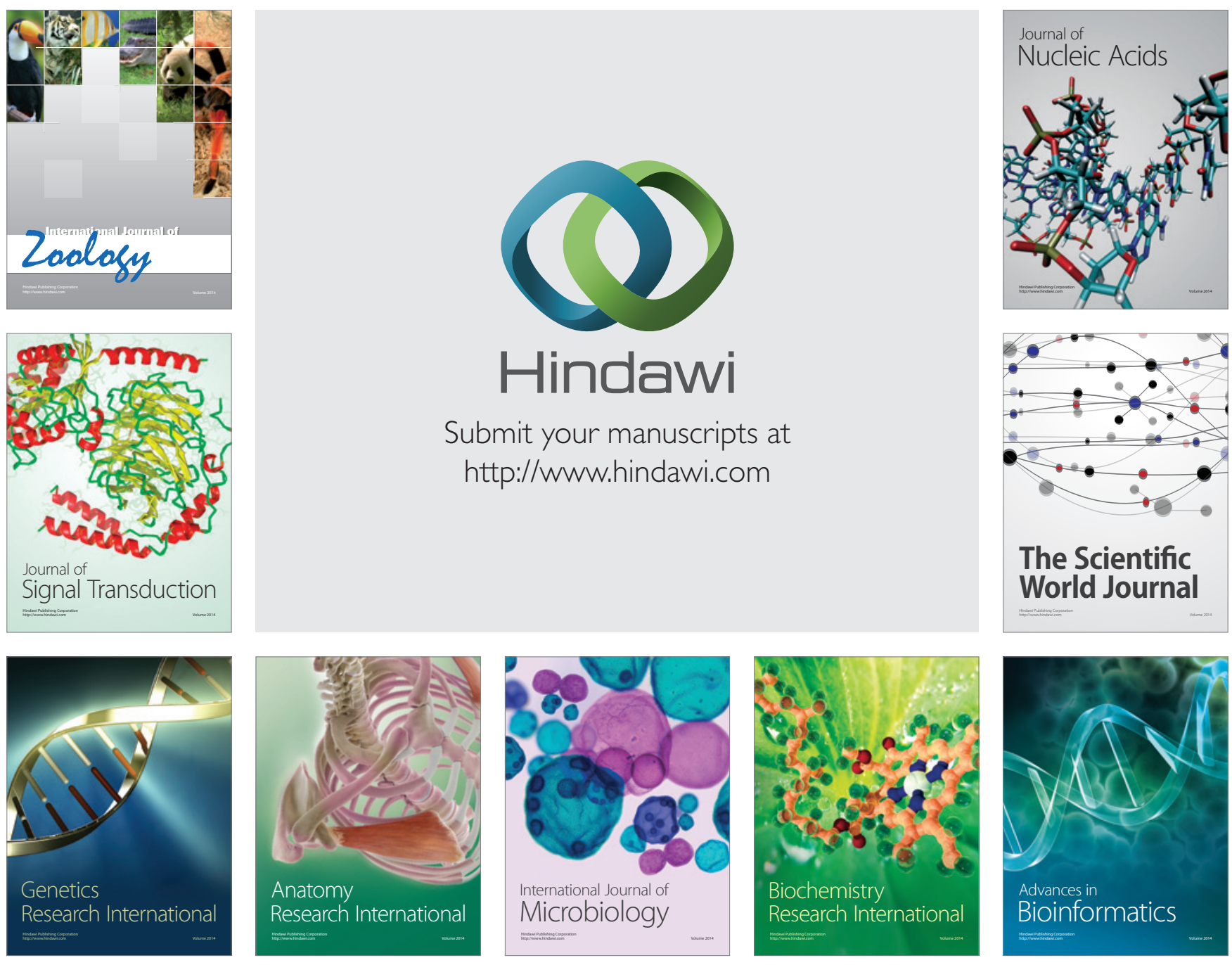

The Scientific World Journal
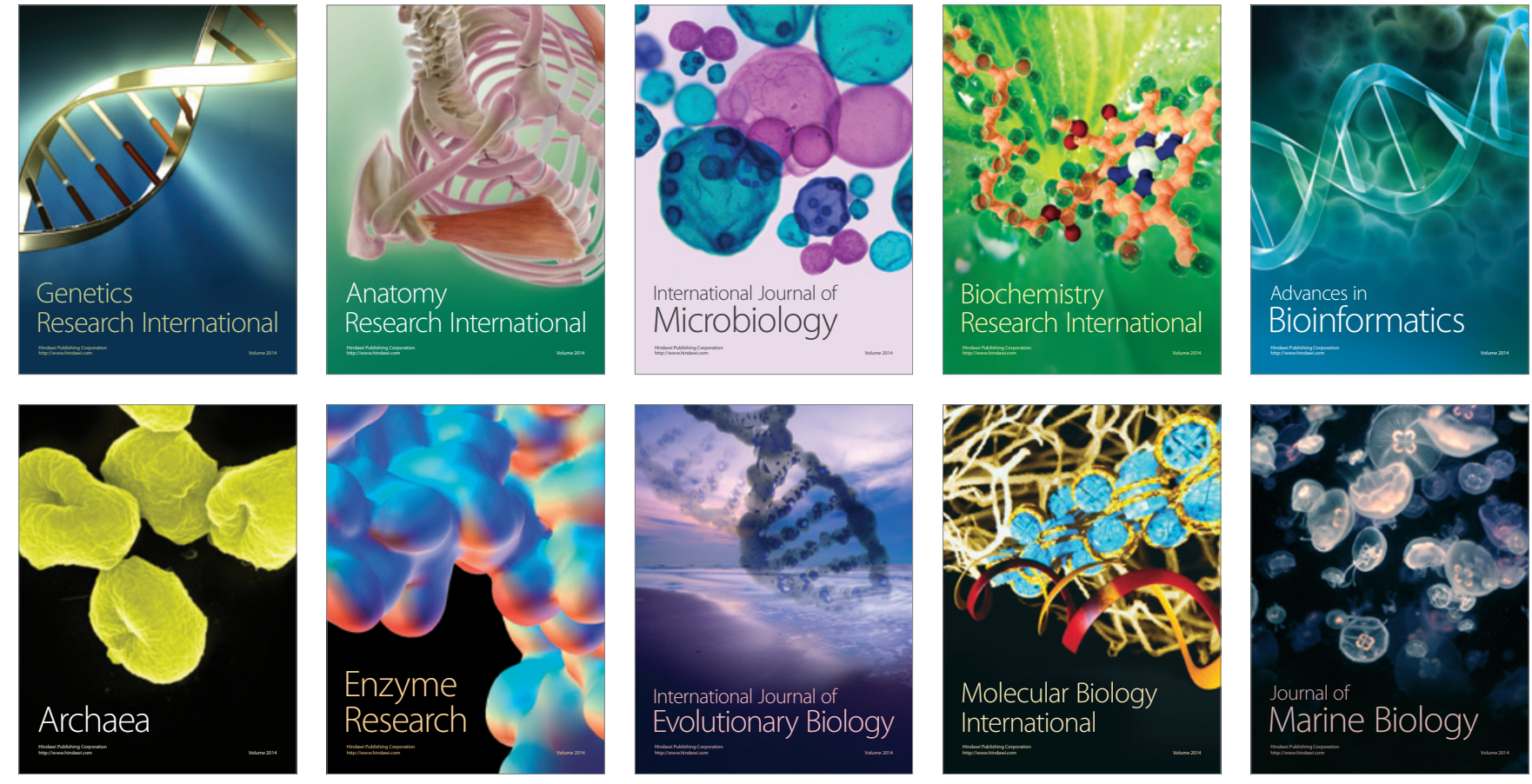